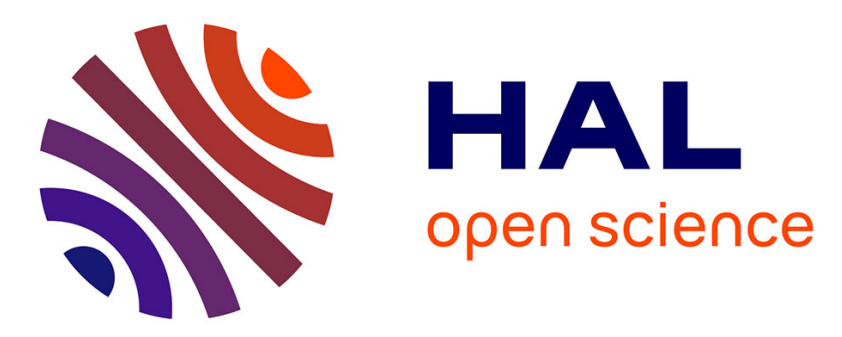

\title{
A mixed adhesion-brittle fracture model and its application to the numerical study of ice shedding mechanisms
}

Lokman Bennani, Philippe Villedieu, Michel Salaün

\section{- To cite this version:}

Lokman Bennani, Philippe Villedieu, Michel Salaün. A mixed adhesion-brittle fracture model and its application to the numerical study of ice shedding mechanisms. Engineering Fracture Mechanics, 2016, vol. 158, pp. 59-80. 10.1016/j.engfracmech.2016.02.050 . hal-01662767

\section{HAL Id: hal-01662767 https://hal.science/hal-01662767}

Submitted on 13 Dec 2017

HAL is a multi-disciplinary open access archive for the deposit and dissemination of scientific research documents, whether they are published or not. The documents may come from teaching and research institutions in France or abroad, or from public or private research centers.
L'archive ouverte pluridisciplinaire HAL, est destinée au dépôt et à la diffusion de documents scientifiques de niveau recherche, publiés ou non, émanant des établissements d'enseignement et de recherche français ou étrangers, des laboratoires publics ou privés. 


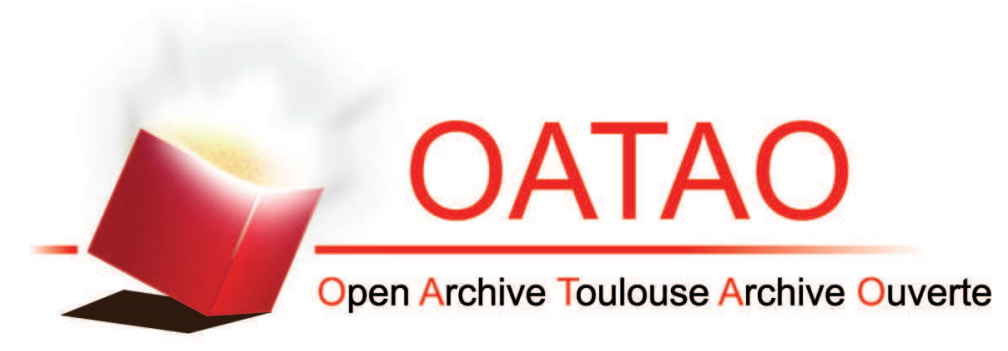

\section{Open Archive Toulouse Archive Ouverte (OATAO)}

OATAO is an open access repository that collects the work of some Toulouse researchers and makes it freely available over the web where possible.

This is an author's version published in: https://oatao.univ-toulouse.fr/18507

Official URL: http://dx.doi.org/10.1016/j.engfracmech.2016.02.050

\section{To cite this version :}

Bennani, Lokman and Villedieu, Philippe and Salaün, Michel A mixed adhesion-brittle fracture model and its application to the numerical study of ice shedding mechanisms. (2016) Engineering Fracture Mechanics, vol. 158. pp. 59-80. ISSN 0013-7944

Any correspondence concerning this service should be sent to the repository administrator: tech-oatao@listes-diff.inp-toulouse.fr 


\title{
A mixed adhesion-brittle fracture model and its application to the numerical study of ice shedding mechanisms
}

\author{
L. Bennani ${ }^{\mathrm{a}, *}$, P. Villedieu ${ }^{\mathrm{b}}$, M. Salaun ${ }^{\mathrm{c}}$ \\ a Institut de Mécanique des Fluides de Toulouse (IMFT) - Université de Toulouse, CNRS-INPT-UPS, Toulouse, France \\ ${ }^{\mathrm{b}}$ ONERA DMAE-MH, 2 Avenue Edouard Belin, 31500 Toulouse, France \\ ${ }^{\mathrm{C}}$ Université de Toulouse, CNRS, ISAE-Supaéro, Institut Clément Ader, 135, avenue de Rangueil, 31077 Toulouse, France
}

\section{Keywords:}

Numerical modelling

Ice shedding

Ice protection

Electro-thermal de-icing

Fracture mechanics

Adhesive debonding

\begin{abstract}
A B S T R A C T
The understanding of ice shedding is of prime importance in the assessment of aeronautical ice protection systems. In this paper, the authors previously studied mechanism is extended to include adhesive debonding. It is based on pressure redistribution in the water film formed at the ice/airfoil interface. The numerical modelling of crack propagation is based on recent work on damage mechanics which provides a general framework. As for adhesive debonding an algebraic model is derived from general mechanical equilibrium relations. Numerical experiments are performed to study an adhesive-debonding/brittlefailure mode detachment, the results of which are discussed.
\end{abstract}

\section{Introduction}

Icing is a major issue in the aeronautical world. Indeed, this phenomenon has plagued powered flight since the beginning of aviation (at which time it was known as the ice problem) [47,46]. Icing is caused by the supercooled water droplets contained in some types of clouds commonly encountered during flight (cumuliform and stratiform) [1]. When these droplets impact the surface of the aircraft their metastable state is broken and they freeze. This therefore leads to ice build-up, which has several undesirable consequences such as a degradation of aerodynamic performances or blocked air intakes. It is therefore one of the most serious threats which may be encountered in the aeronautical world.

Hence, manufacturers are required to ensure the airworthiness of their aircrafts by complying with strict certifications and regulations. To do so, many ice protection systems have been developed over the years. Today, in the context of more electric aircrafts and the reduction of energy consumption, the electro-thermal ice protection system (ETIPS) is being considered as a promising technology.

This system is composed of electrical heater installed within a multi-layered material and can be used in anti-icing or deicing configurations [39,43]. The nominal operation of an ETIPS is illustrated in Fig. 1. It first involves what is called the 'parting strip' (heater C). This element is placed near the leading edge and is always active when icing conditions are encountered. This has the effect of keeping the leading edge clean from ice. The other heaters are activated according to a given power cycle. Ice is therefore able to build up in their neighbourhood. When a given heater is activated, the generated heat melts

\footnotetext{
* Corresponding author.

E-mail addresses: lokman.bennani@imft.fr (L. Bennani), philippe.villedieu@onera.fr (P. Villedieu), m.salaun@isae.fr (M. Salaun).
} 


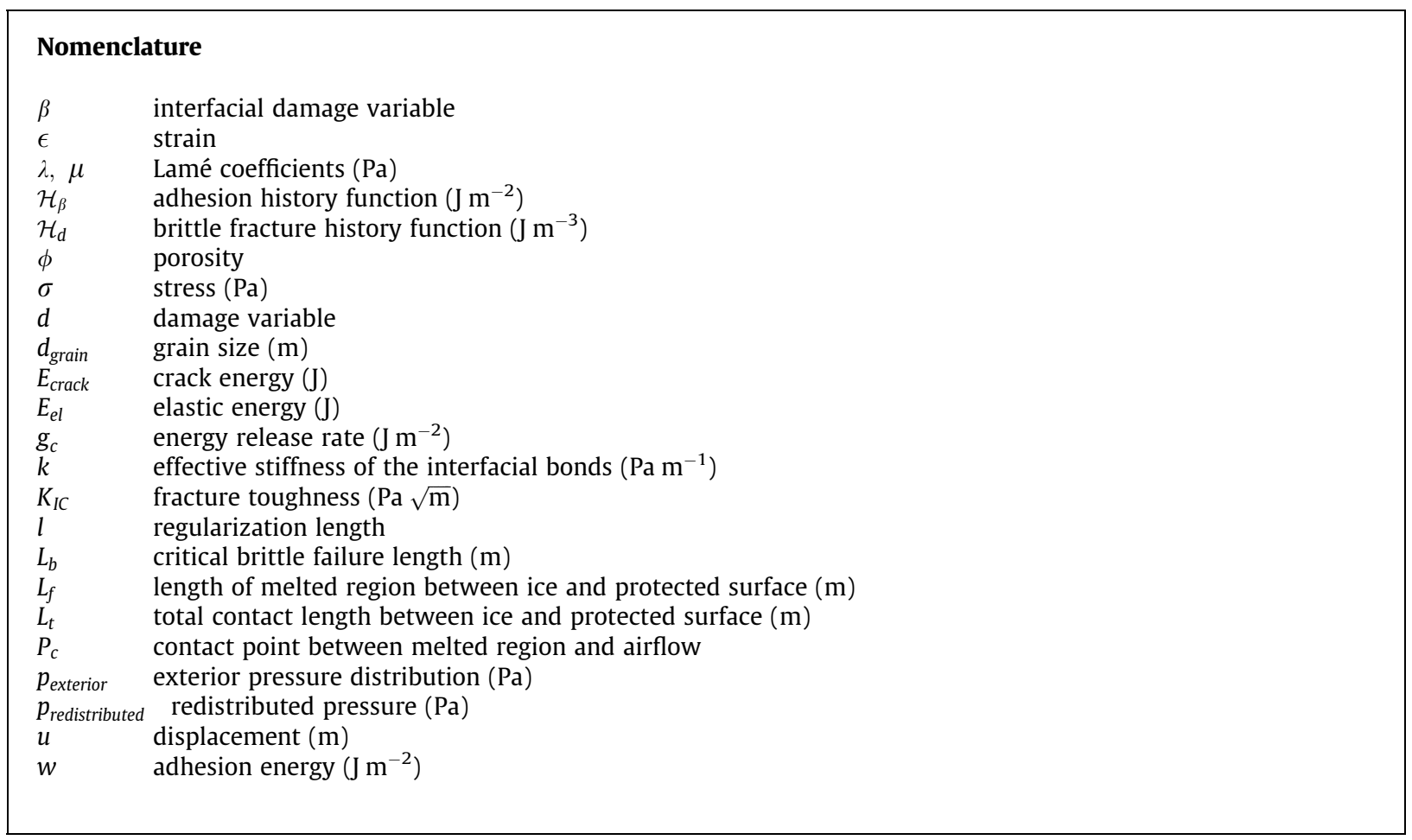

the ice at the interface. The ice is then less able to stay attached to the surface and is eventually shed by the effect of the aerodynamic forces.

The understanding of ice shedding is of prime importance in the assessment of the performance of an ETIPS. It is essential to understand by which mechanisms the aerodynamic forces are able to detach the ice. However, very few studies exist and ice shedding is to this day still not well understood. Experimental studies dealing with the precise mechanisms of ice shedding are almost inexistent and present extremely scattered results [5,41].

On the numerical side, Scavuzzo et al. performed a finite element analysis of the stress distribution due to aerodynamic forces in an accreted ice block [40]. More recently, Zhang et al. have used a crack propagation and re-meshing technique to study ice break up [2]. However these studies did not take into account the effect of an ice protection system.

From a practical point of view, ice shedding is taken into account in state of the art icing codes by using a highly empirical criterion. The criterion works by comparing the length of the liquid water film formed at the interface with the whole contact length. It states that, if the ratio of these two lengths exceeds a user defined value (typically $80 \%$ of the whole contact length), then the ice block detaches [39]. However, such an empirical criterion is unsatisfactory. Therefore, to obtain more physical ice shedding models, a better understanding of the detachment process is needed.

In this paper, the authors build upon their previous study [24] by incorporating new possible debonding mechanisms. First, the proposed detachment mechanisms are presented. Then, the modelling and numerical techniques used in this study are introduced. This will be followed by a parameter identification after which numerical experiments are performed. Finally the results are discussed.

As shall be stressed out later, it is extremely difficult to characterize the mechanical properties of atmospheric ice. Hence, it should be noted that the goal here is not to provide highly accurate predictions. It is rather to propose a general methodology which can be used to explore and explain different ice shedding mechanisms in a qualitative way, opening the door to their experimental investigation. The presented methodology is moreover general enough to be applied to the study of other problems. In addition, this approach has the advantage of locally predicting the initiation of detachment or fracture.

With this approach, qualitative progress is sought on the comprehension of the physical mechanisms at play, in particular concerning the interplay between adhesive detachment and brittle failure. Indeed, this aspect is of great importance to the design of electro-thermal ice protection systems (heating cycles, choice of materials, etc.).

\section{Proposed mechanism}

Let us consider the following situation (illustrated in Fig. 2). In the nominal functioning mode, ice has built up just aft the parting strip. In due time, the corresponding heater will be activated. This will lead to the creation of a liquid water film, extending over a distance $L_{f}$, between the surface and the ice. Due to hydrostatic equilibrium, the pressure exerted by the 


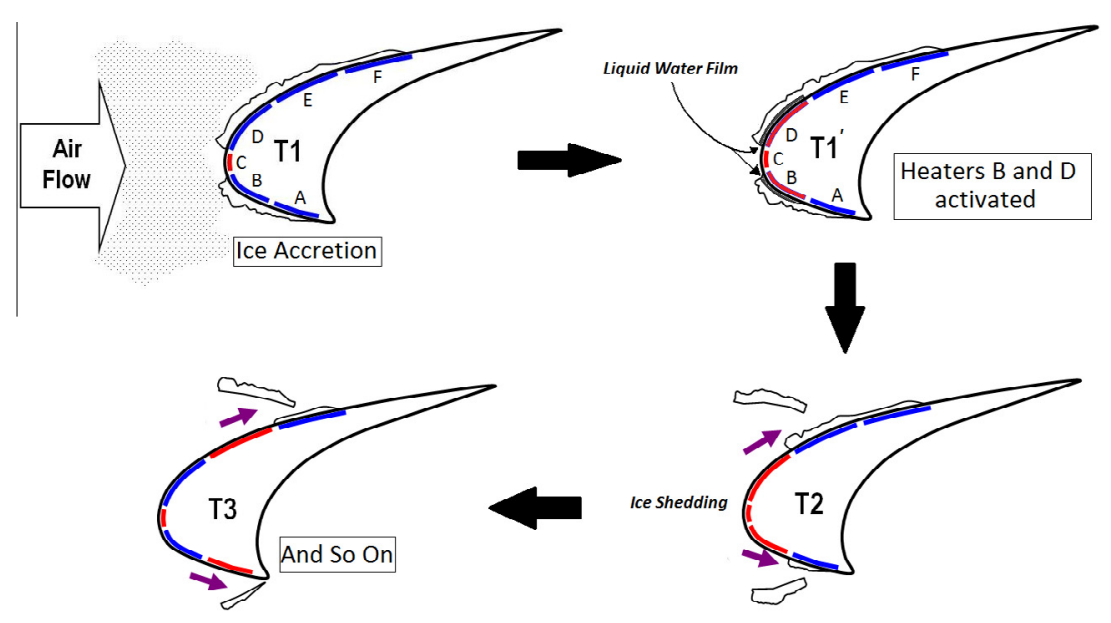

Fig. 1. Operation of an ETIPS.

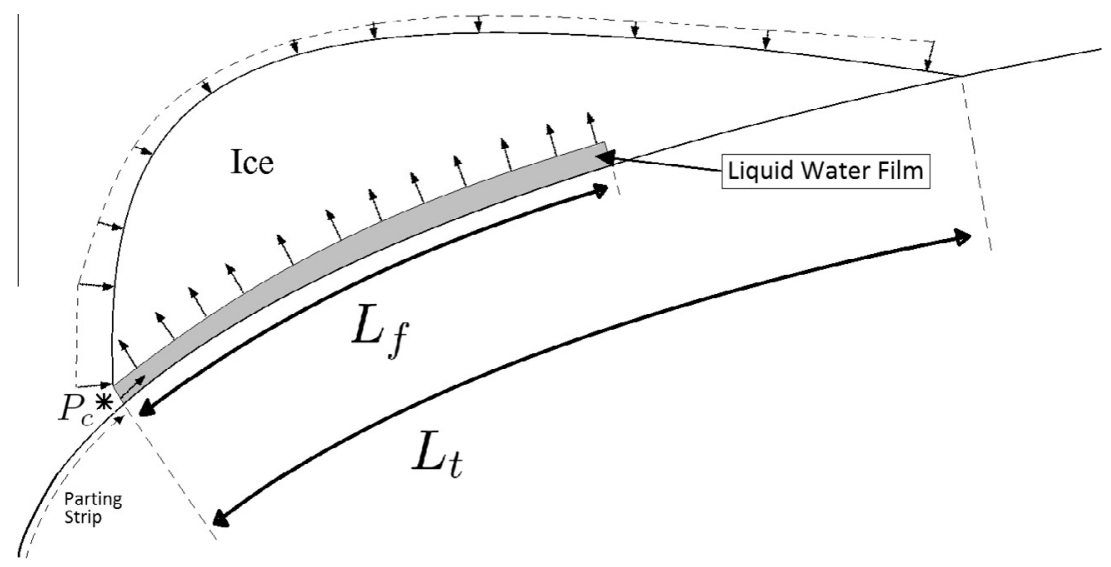

Fig. 2. Illustration and schematic pressure distribution.

aerodynamic flow field at $P_{c}$ will be redistributed within the film (assuming the film is static ${ }^{1}$ and negligible surface tension effects which are discussed in Section 5.4). Moreover, the flow over the lump of ice will induce a pressure decrease, meaning that the pressure recovered in the film will be higher than that acting on the external surface.

This pressure distribution creates a lifting force. To this force, one has to add the viscous forces, which are tangential. Thanks to these forces, several outcomes may be possible (see Figs. 3-6):

- The whole length is melted $\left(L_{f}=L_{t}\right)$ in which case the ice no longer adheres to the surface (or only by means of surface tension effects).

- Adhesive interfacial debonding: part of the length $L_{f}=x \% L_{t}$ is melted and the adhesion forces that hold ice on the surface are no longer strong enough.

- Cohesive interfacial debonding: part of the length $L_{f}=x \% L_{t}$ is melted, ice can still adhere, but a crack may nucleate due to stress concentration and propagate along the interface.

- Bulk failure: part of the length $L_{f}=x \% L_{t}$ is melted, ice can still adhere, but a crack may nucleate due to stress concentration and propagate inside the bulk of the ice block, therefore tearing off a part of the ice.

- Ice shedding is due to an interplay of all or part of the previous mechanisms, as the experiments of Wei et al. seem to suggest [48].

\footnotetext{
${ }^{1}$ In fact, as liquid water takes up less volume than ice, a gap may form in the melted region. That is to say, the water film may not entirely occupy the volume formerly made out of ice and air may be allowed to fill in the gap. However, we would still be in a case of hydrostatic pressure equilibrium. Therefore we would still have pressure redistribution as described.
} 


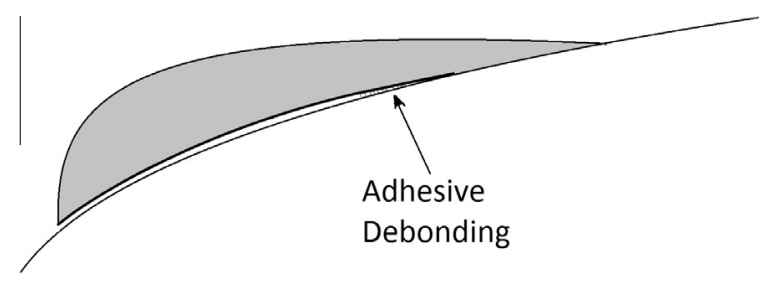

Fig. 3. Adhesive debonding.

In this paper, a mixed adhesive debonding/brittle fracture ice shedding mechanism is investigated, extending the authors previous work which considered only brittle failure.

\section{Mechanical modelling}

In this study, a framework close to continuum damage mechanics is chosen. In the following paragraphs the brittle failure and adhesive debonding models are presented.

\subsection{Brittle failure model}

The brittle failure model which is used for this study relies strongly on the variational approach to fracture [18]. It is based on a principle of conservation of energy which translates the interplay between elastic deformation energy and crack surface energy [3]. Fig. 7 shows an illustration of a cracked material.

The crack energy is obtained by introducing a damage variable $d$ and using a regularized crack energy functional (see for example Bourdin et al. [8]).

$$
E_{\text {crack }}=\int_{\Omega} \phi(d, \nabla d) d V=\int_{\Omega} g_{c}\left[\frac{1}{2 l} d^{2}+\frac{l}{2} \nabla d \cdot \nabla d\right] d V
$$

where $g_{c}$ is the crack energy release rate, the damage variable $d$ lies between 0 and $1(d(x)=0$ corresponding to an undamaged state and $d(x)=1$ to a fractured state). $l$ is a regularization length which is chosen according to the recommendations of the literature [13] and tests performed in previous work [24]. The values chosen in this work are given in Table A.4.

On the other hand, following Miehe et al. [13], the elastic energy is split into purely tensile and compressive parts defined by using the eigenvalues of $\epsilon\left(\epsilon_{1}\right.$ and $\epsilon_{2}$ in two dimension $)$ and a positive/negative part function noted $\langle\cdot\rangle_{ \pm}$:

$$
\begin{aligned}
& \psi_{0}^{+}(\epsilon)=\frac{\lambda}{2}\left\langle\epsilon_{1}+\epsilon_{2}\right\rangle_{+}^{2}+\mu\left(\left\langle\epsilon_{1}\right\rangle_{+}^{2}+\left\langle\epsilon_{2}\right\rangle_{+}^{2}\right) \\
& \psi_{0}^{-}(\epsilon)=\frac{\lambda}{2}\left\langle\epsilon_{1}+\epsilon_{2}\right\rangle_{-}^{2}+\mu\left(\left\langle\epsilon_{1}\right\rangle_{-}^{2}+\left\langle\epsilon_{2}\right\rangle_{-}^{2}\right)
\end{aligned}
$$

$\psi_{0}^{+}$and $\psi_{0}^{-}$represent respectively the undamaged tensile and compressive elastic energies. Thus only $\psi_{0}^{+}(\epsilon)$, the tensile part, is multiplied by a function of $d$, noted $f(d)$. The function $f(d)$ represents the degradation of tensile energy due to crack formation and is chosen accordingly [13].

$$
E_{e l}=\int_{\Omega} \phi(d, \nabla d) d V=\int_{\Omega}\left[f(d) \psi_{0}^{+}(\epsilon)+\psi_{0}^{-}(\epsilon)\right] d V
$$

Then, using variational arguments, one may show that:

$$
\begin{array}{ll}
-\operatorname{div}(\sigma(u, d))=f_{\text {vol }} & \text { in } \Omega \\
\sigma \cdot n=f_{\text {surf }} & \text { on } \Gamma_{1} \\
u=u_{d} & \text { on } \Gamma_{2} \\
\frac{g_{c}}{l} d-g_{c} l \triangle d=2(1-d) \psi_{0}^{+}(\epsilon) & \text { in } \Omega \\
\nabla d \cdot n=0 & \text { on } \partial \Omega
\end{array}
$$

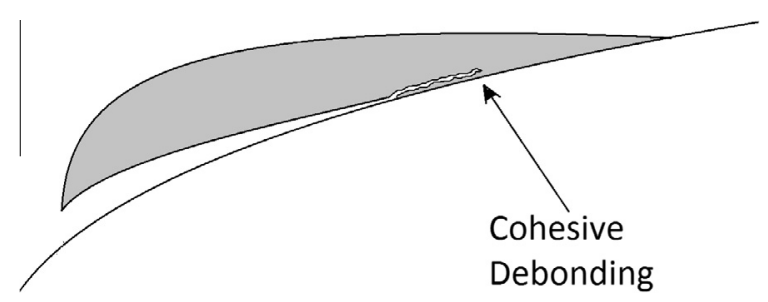

Fig. 4. Cohesive debonding. 


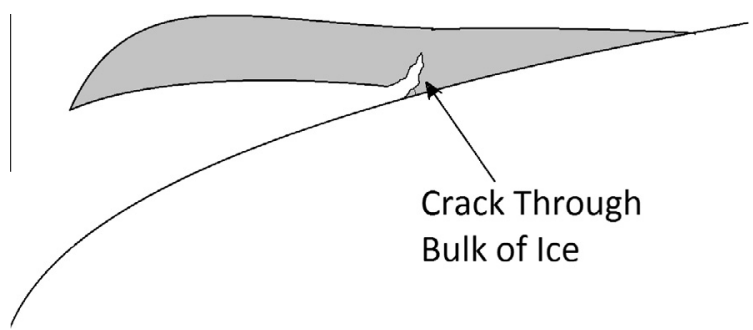

Fig. 5. Bulk failure.

where $\sigma$ is the stress tensor and is deduced from the elastic energy: $\sigma=\frac{\partial \psi}{\partial \epsilon}$.

Eq. (5) are nonlinear and describe a stationary damaged equilibrium state compatible with the external constraints. They translate conservation of energy. In order to incorporate the irreversible aspect of crack propagation, Miehe et al. [13] introduce a history function $\mathcal{H}_{d}$. The following iterative algorithm, which is implemented using a finite element method [24], is inspired by the algorithm proposed by Miehe et al. [13].

- Compute the history field:

$$
\mathcal{H}_{d}^{i}=\max \left(\mathcal{H}_{d}^{i-1}, \psi_{0}^{+}\left(\epsilon^{i-1}\right)\right)
$$

- Compute the damage field:

$$
\begin{array}{r}
\frac{g_{c}}{l} d^{i}-g_{c} l \Delta d^{i}=2\left(1-d^{i}\right) \mathcal{H}_{d}^{i} \text { in } \Omega \\
\nabla d^{i} \cdot n=0 \text { on } \partial \Omega
\end{array}
$$

- Compute the displacement field:

$$
\begin{array}{r}
-\operatorname{div}\left(\sigma^{i}\left(\epsilon^{i}, d^{i}\right)\right)=f_{\text {vol }} \text { in } \Omega \\
\sigma^{i} \cdot n=f_{\text {surf }} \text { on } \Gamma_{1} \\
u^{i}=u_{d} \text { on } \Gamma_{2}
\end{array}
$$

The main advantage of this method is that it does not require an initial crack. On the other hand, it should be noted that the main drawback is the need of a refined mesh. Especially in the case of imposed boundary load, the crack tends to spread if the mesh is not refined enough [12,24].

Before we proceed to the next section describing the adhesion model, it seems important to outline how the stress tensor is computed. The stress tensor $\sigma$ derives from the definition of the elastic energy and takes the following form:

$$
\sigma=\frac{\partial \psi}{\partial \epsilon}=f(d)\left[\lambda\langle\operatorname{tr}(\epsilon)\rangle_{+} \mathbb{1}+2 \mu \epsilon_{+}\right]+\left[\lambda\langle\operatorname{tr}(\epsilon)\rangle_{-} \mathbb{1}+2 \mu \epsilon_{-}\right]
$$

The problem here is that stress and strain are not linked by a linear constitutive law. The definition above involves non linear functions such as the positive part $\langle\cdot\rangle_{+}$. In particular, the computation of the positive/negative part of $\epsilon$ requires projection operators which depend on the current local value of the strain tensor. Moreover, note that the volumetric deformation term $\operatorname{tr}(\epsilon)$ is also split into distinct contributions $\left(\langle\operatorname{tr}(\epsilon)\rangle_{ \pm}\right)$to tensile and compressive energies. Hence, on the whole, the mechanical equilibrium problem remains strongly non-linear.

In order to linearize this problem, a method which takes advantage of the iterative approach to solving the global problem is used. Indeed, at any given iteration $i$ of the global problem, the strain tensor at iteration $i-1$ is known. Therefore, it is $\epsilon^{i-1}$

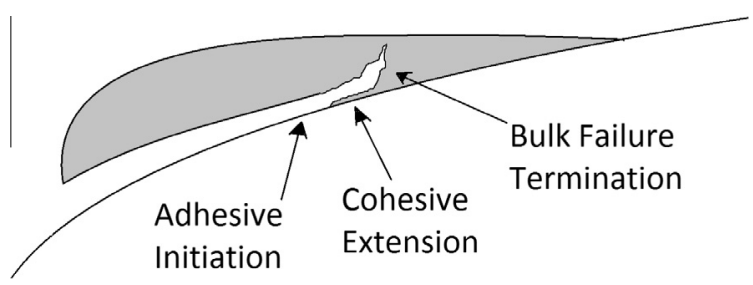

Fig. 6. Adhesive initiation followed by cohesive extension. Process terminates with bulk failure. 


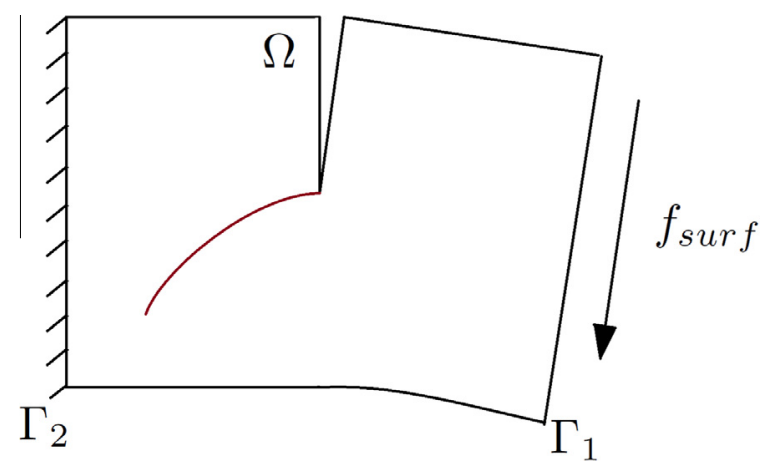

Fig. 7. Illustration of a cracked material.

which is used in order to compute the projection operators. Therefore, the eigenvalues and eigenvectors used to compute the various objects relative to the negative/positive decomposition are those of $\epsilon^{i-1}$. More details are given in Appendix B.

\subsection{Adhesive debonding model}

Adhesion modelling is also an active area of research (see for example [32,34,31]). Here, the approach initiated by Frémond [17] is used as a starting point. An algebraic model is then deduced and an algorithm inspired by that used for brittle failure is used.

Let us consider a block of material adhering to a given surface, as depicted in Fig. 8. A surface force $T$ is applied on the upper boundary, $\Gamma_{1}$. In reaction to that force, the bonds at the bottom interface, noted $\Gamma_{2}$ exert a force $R$ on the block of material. The force $R$ tends to counteract $T$ and to maintain the block attached to $\Gamma_{2}$.

The mechanical equilibrium of such a configuration is described by the following equations:

$$
\begin{array}{r}
-\operatorname{div}(\sigma)=f_{v o l} \text { in } \Omega \\
\sigma \cdot n=T \text { on } \Gamma_{1} \\
\sigma \cdot n=R \text { on } \Gamma_{2}
\end{array}
$$

Note that later on, it will be shown that (14) takes the form of a kind of Fourier-Robin condition, hence ensuring wellposedness.

The idea, as in the brittle failure model, is to introduce an adhesive damage variable, noted $\beta$. This variable represents the state of the microscopic bonds that maintain the block attached to the interface through the force $R$. Like $d$, the variable $\beta$ is defined to vary from 0 for a fully bonded state to 1 for a fully debonded state.

The total energy is then defined as the sum of the elastic energy of the block of material, the energy stored in the microscopic bonds and the surface energy which appears when debonding occurs (creation of new interfaces). Energy densities are then introduced: $\psi(\epsilon)$ for the elastic bulk energy, $\phi(\beta)$ for surface energy and $\gamma(\beta, u)$ for the energy stored inside the microscopic bonds. The choice of the inputs of the energies $\phi$ and $\gamma$ can be justified on intuitive physical grounds.

$\phi$ is the energy linked to the amount of surface created during the debonding process. This amount of surface is directly linked to $\beta$, which represents the broken or unbroken state of the microscopic bonds. Surface energy will be associated with regions where the bonds are broken. Hence it is natural to consider $\phi$ as a function of $\beta$.

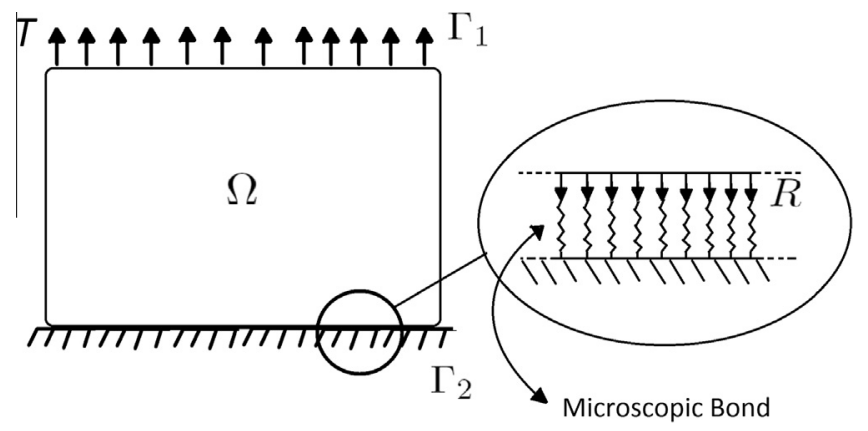

Fig. 8. Illustration of a solid body adhering to a surface. 
$\gamma$ is the energy stored within the microscopic bonds. First, the more $\beta$ will approach the value 1 , the weaker the bonds will be and the less energy they will be able to store. Secondly, for a given local $\beta$, the amount of energy stored within the bonds will be a function of the displacement $u$ applied on the bonds. The bonds may be thought of as springs and indeed this visualization is coherent with the model which will be derived and the choice of the energy $\gamma$.

Hence, the total energy is defined as

$$
E_{\text {tot }}=\int_{\Omega} \psi(\epsilon) d V+\int_{\Gamma_{2}} \phi(\beta) d \Gamma+\int_{\Gamma_{2}} \gamma(\beta, u) d \Gamma
$$

Therefore, the variation in total energy reads:

$$
\delta E_{\text {tot }}=\int_{\Omega} \frac{\partial \psi}{\partial \epsilon}: \delta \epsilon d V+\int_{\Gamma_{2}} \frac{\partial \phi}{\partial \beta} \delta \beta d \Gamma+\int_{\Gamma_{2}}\left[\frac{\partial \gamma}{\partial \beta} \delta \beta+\frac{\partial \gamma}{\partial u} \delta u\right] d \Gamma
$$

Moreover, the variation of total energy must be equal to the work performed by external forces:

$$
\delta E_{\text {tot }}=W_{\text {ext }}
$$

Here, this work must take into account the forces acting on the whole boundary of the block of material as well as the forces acting upon the microscopic bonds (as their energy is accounted for in the total energy). Let us note $f_{\text {ext }}$ the forces acting on the boundary of $\Omega$ and $f_{\text {bond }}$ the force exerted by the material block on the microscopic bonds. The work $W_{\text {ext }}$ can therefore be written as follows:

$$
W_{\text {ext }}=\int_{\partial \Omega} f_{\text {ext }} \delta u d \Gamma+\int_{\Gamma_{1}} f_{\text {bond }} \delta u d \Gamma
$$

By the action/reaction principle, the force $f_{\text {bond }}$ is equal to $-R$. By combining Eqs. (15) and (16) and using the mechanical equilibrium equations, one obtains the following:

$$
\int_{\Omega}\left[\frac{\partial \psi}{\partial \epsilon}-\sigma\right]: \delta \epsilon d V+\int_{\Gamma_{2}}\left[\frac{\partial \phi}{\partial \beta}+\frac{\partial \gamma}{\partial \beta}\right] \delta \beta d \Gamma+\int_{\Gamma_{2}}\left[\frac{\partial \gamma}{\partial u}+R\right] \delta u d \Gamma=0
$$

Eq. (17) has to hold for every variation $\delta \beta, \delta u, \delta \epsilon$, therefore:

$$
\begin{gathered}
\sigma=\frac{\partial \psi}{\partial \epsilon} \text { in } \Omega \\
\frac{\partial \phi}{\partial \beta}=-\frac{\partial \gamma}{\partial \beta} \text { on } \Gamma_{2} \\
R=-\frac{\partial \gamma}{\partial u} \text { on } \Gamma_{2}
\end{gathered}
$$

As in Section 3.1, the choice of the elastic bulk energy density determines the stress tensor. It may be chosen in its classic linear elastic form to yield Hooke's law. However, in this study, as the adhesion model will be coupled with the brittle failure model, the elastic bulk energy is defined as in Section 3.1 by splitting into tensile and compressive parts.

The density $\phi$ represents the surface energy created in the debonding process. By analogy with the bulk damage model, it is defined as follows:

$$
\phi=w \beta^{2}
$$

where $w$ is the adhesion energy per unit area. Note that as the subsequent computations are performed in a two dimensional framework, the debonding naturally follows the boundary line and gradient terms would not be of fundamental use. Hence they are not introduced.

Finally, $\gamma$ is the density of energy stored inside the interfacial microscopic bonds. Inspired by the fracture modelling approach, we choose here to split the contributions of the interfacial displacement using its normal ( $\left.u_{n}\right)$ and tangential $\left(u_{\tau}\right)$ components. At this point, it is important to note that the term $-\frac{\partial \gamma}{\partial \beta}$ will be the driving force of the debonding process. It seems natural to consider that $u_{n}$ will only contribute to the debonding process if it is positive and that $u_{\tau}$ will always do. Moreover, this contribution has to degrade itself with increasing $\beta$. Therefore, the following expression is chosen for $\gamma$ :

$$
\gamma=f(\beta) \frac{k}{2}\left[\left\langle u_{n}\right\rangle_{+}^{2}+\alpha_{\tau} u_{\tau}^{2}\right]+\frac{k}{2}\left\langle u_{n}\right\rangle_{-}^{2}
$$

where $f$ is the same function here as for the brittle failure model, that is to say $f(\beta)=(1-\beta)^{2}$ and $k$ is an effective stiffness parameter. $\alpha_{\tau}$ is a parameter representing the contribution of the tangential displacement, which could be different from that of the normal one. $u_{n}$ and $u_{\tau}$ are linked to $u_{x}$ and $u_{y}$ through the following relation:

$$
\left(\begin{array}{l}
u_{\tau} \\
u_{n}
\end{array}\right)=\left(\begin{array}{cc}
\cos (\theta) & \sin (\theta) \\
-\sin (\theta) & \cos (\theta)
\end{array}\right)\left(\begin{array}{l}
u_{x} \\
u_{y}
\end{array}\right)
$$


where $\theta$ is the angle between the tangent to $\Gamma_{2}$ and the $x$ axis, as shown in Fig. 9 .

For simplicity, the more compact notation is introduced for $\gamma$ :

$$
\gamma=f(\beta) \gamma_{\beta}+\gamma_{0}
$$

where

$$
\gamma_{\beta}=\frac{k}{2}\left[\left\langle u_{n}\right\rangle_{+}^{2}+\alpha_{\tau} u_{\tau}^{2}\right]
$$

and

$$
\gamma_{0}=\frac{k}{2}\left\langle u_{n}\right\rangle_{-}^{2}
$$

With such choices, the equation defining the local damage state of the interfacial elastic bonds (19) reduces to the following algebraic relation:

$$
\frac{\partial \phi}{\partial \beta}=-\frac{\partial \gamma}{\partial \beta} \rightarrow w \beta=(1-\beta) \frac{k}{2}\left[\left\langle u_{n}\right\rangle_{+}^{2}+\alpha_{\tau} u_{\tau}^{2}\right]=(1-\beta) \gamma_{\beta}
$$

leading to the following formula for $\beta$ :

$$
\beta=\frac{\gamma_{\beta}}{w+\gamma_{\beta}}
$$

The choice of $\gamma$ also defines the reaction force $R$ through its partial derivatives with respect to $u_{x}$ and $u_{y}$. Hence the partial derivatives of $\left\langle u_{n}\right\rangle_{+}^{2},\left\langle u_{n}\right\rangle_{-}^{2}$ and $u_{\tau}^{2}$ need to be computed. Starting with $\left\langle u_{n}\right\rangle_{+}^{2}$ :

$$
\frac{\partial\left\langle u_{n}\right\rangle_{+}^{2}}{\partial u_{x}}=\frac{\partial\left\langle u_{n}\right\rangle_{+}^{2}}{\partial u_{n}} \frac{\partial u_{n}}{\partial u_{x}}=2\left\langle u_{n}\right\rangle_{+}
$$

So as to express $\left\langle u_{n}\right\rangle_{+}$explicitly in terms of $u$, the following function is introduced:

$$
\delta_{+}= \begin{cases}0 & \text { if } u_{n}<0 \\ 1 & \text { if } u_{n} \geqslant 0\end{cases}
$$

which leads to the expression:

$$
\left\langle u_{n}\right\rangle_{+}=\delta_{+} u_{n}=\delta_{+}[-\sin (\theta) \cos (\theta)] u
$$

Noting that the partial derivative of $u_{n}$ with respect to $u_{x}$ is simply:

$$
\frac{\partial u_{n}}{\partial u_{x}}=-\sin (\theta)
$$

the partial derivative of $\left\langle u_{n}\right\rangle_{+}^{2}$ with respect to $u$ reads:

$$
\frac{\partial\left\langle u_{n}\right\rangle_{+}^{2}}{\partial u_{x}}=-2 \sin (\theta) \delta_{+}[-\sin (\theta) \cos (\theta)] u
$$

which finally yields the following expression:

$$
\frac{\partial\left\langle u_{n}\right\rangle_{+}^{2}}{\partial u}=2 \delta_{+}\left(\begin{array}{cc}
\sin ^{2}(\theta) & -\sin (\theta) \cos (\theta) \\
-\sin (\theta) \cos (\theta) & \cos ^{2}(\theta)
\end{array}\right)\left(\begin{array}{l}
u_{x} \\
u_{y}
\end{array}\right)
$$

The partial derivatives of $\left\langle u_{n}\right\rangle_{-}^{2}$ and $u_{\tau}^{2}$ follow exactly the same straightforward procedure (with the introduction of a function $\delta_{-}$for $\left\langle u_{n}\right\rangle_{-}$). Therefore, the reaction force $R$ is given by:

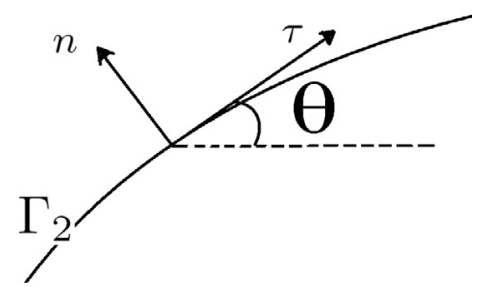

Fig. 9. Definition of the angle $\theta$. 


$$
R=-\frac{\partial \gamma}{\partial u}=-k F(\beta, u) u
$$

where $F(\beta, u)$ is a matrix given by

$$
\begin{aligned}
F(\beta, u)= & f(\beta) \delta_{+}\left(\begin{array}{cc}
\sin ^{2}(\theta) & -\sin (\theta) \cos (\theta) \\
-\sin (\theta) \cos (\theta) & \cos ^{2}(\theta)
\end{array}\right)+\alpha_{\tau} f(\beta)\left(\begin{array}{cc}
\cos ^{2}(\theta) & \sin (\theta) \cos (\theta) \\
\sin (\theta) \cos (\theta) & \sin ^{2}(\theta)
\end{array}\right) \\
& +\delta_{-}\left(\begin{array}{cc}
\sin ^{2}(\theta) & -\sin (\theta) \cos (\theta) \\
-\sin (\theta) \cos (\theta) & \cos ^{2}(\theta)
\end{array}\right)
\end{aligned}
$$

It is interesting to note that this will lead to a kind of Fourier-Robin boundary condition on $\Gamma_{2}$.

As a final step, inspired by the method used for the brittle failure model and considering that the debonding process is also irreversible, a history function, noted $\mathcal{H}_{\beta}$, is introduced and replaces $\gamma_{\beta}$ in Eq. (23). This leads to the formulation of the following algorithm:

- Compute the history field:

$$
\mathcal{H}_{\beta}^{i}=\max \left(\mathcal{H}_{\beta}^{i-1}, \gamma_{\beta}\left(u^{i-1}\right)\right)
$$

- Compute the interfacial damage field:

$$
\beta^{i}=\frac{\mathcal{H}_{\beta}^{i}}{w+\mathcal{H}_{\beta}^{i}} \quad \text { on } \Gamma_{2}
$$

- Compute the displacement field:

$$
\begin{array}{r}
-\operatorname{div}\left(\sigma^{i}\left(\epsilon^{i}\right)\right)=f_{v o l} \text { in } \Omega \\
\sigma^{i} \cdot n=T \text { on } \Gamma_{1} \\
\sigma^{i} \cdot n+k F\left(\beta^{i}, u^{i-1}\right) u^{i}=0 \text { on } \Gamma_{2}
\end{array}
$$

It should be noted that the Fourier-Robin boundary condition is linearized by taking $u^{i-1}$ as the displacement defining $F$.

\subsection{Coupled adhesive/brittle failure algorithm}

In order to perform a mixed adhesive/brittle failure computation, the two previously described methods are combined in the following way:

Algorithm 1. Adhesive/brittle failure computation

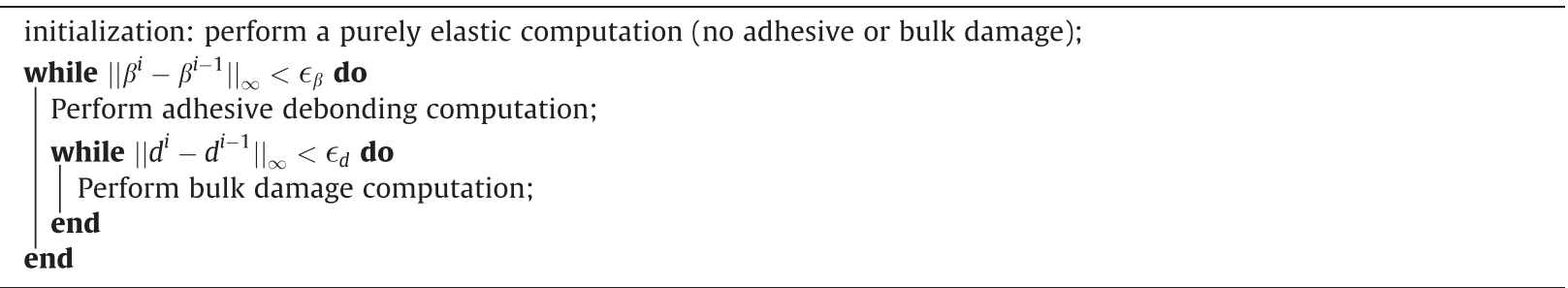

where $\epsilon_{\beta}$ and $\epsilon_{d}$ are set to $10^{-3}$.

The algorithm can be viewed in the following manner. For each debonded configuration, a bulk damage computation is performed to check whether no corresponding compatible bulk failure state exists. Bulk damage and interfacial damage interact through the boundary conditions. Indeed, a increase in interfacial damage $\beta$ will change the boundary conditions. In turn this change will impact the deformed state of the material, from which is deduced bulk damage. The convergence loops are performed in this order as, in the case studied here at least, adhesive debonding is the initiating process and bulk failure is the terminating one (as shall be shown in the following sections). Therefore, the loops are performed in this order to be physically consistent with the application at hand. Combining both models in a more general coupled framework is the focus of current work.

In order to solve the previously presented equations, a finite element procedure is implemented using Lagrange P1 shape functions. The resulting linear systems are solved using a direct solver based on the LU decomposition. More details on the numerical method, especially concerning the boundedness of the damage variables, can be found in Appendix C. 


\section{Mechanical properties of atmospheric ice}

One of the main problems that arises is to determine what mechanical properties are going to be used in order to characterize atmospheric ice. Unfortunately, very few studies on the subject exist. Most studies are interested in the tensile or compressive strength but do not provide many information on mechanical characteristics in the form of well defined laws $[22,29,7]$. These experiments are very difficult to conduct due to the vast number of parameters on which those properties depend, making the issue all the more complicated.

Young's modulus for atmospheric ice was recently estimated by Eskandarian using poroelasticity and Hill's averaging method. The reported values are around 9.5 MPa [26]. Kermani also attempted to measure Young's modulus by static loading using an extensometer but found values around $5 \mathrm{MPa}$. He pointed out that these measurements were less accurate giving several sources of error [28].

\subsection{Elastic and fracture toughness properties}

Concerning the elastic properties, a combination of empirical laws based on information gathered by Schulson and Duval [15] and proposed by the authors in their previous work [24] will be used.

The parameters characterizing homogeneous isotropic elastic behaviour of polycrystalline ice are given in Table 1 [15,36]:

Then, the following empirical law is used to define a more general behaviour through Young's modulus:

$$
E=E\left(T_{r}\right)\left[1-a\left(T-T_{r}\right)\right]-b \phi_{p} \quad \phi_{p} \leqslant 0.1 \text { and } \mathrm{E} \text { is in GPa }
$$

where $\phi_{p}$ is the porosity, $d_{\text {grain }}$ the grain size, $a=1.42 \times 10^{-3} \mathrm{~K}^{-1}, b=35.1 \mathrm{GPa}$ and $T_{r}$ is the temperature at which the initial measurement was conducted.

Concerning fracture, as noted by Petrovic, it has not been intensively investigated [37]. The results are scattered around the values of $80-140 \mathrm{kPa} \mathrm{m}^{1 / 2}$ and no clear trend seems to be apparent. Here, as the following numerical simulations are set for a free stream temperature of $-10^{\circ} \mathrm{C}$, the fracture toughness is set to be the average of the measurements performed by Kermani at that temperature: $K_{I C}=110 \mathrm{kPa} \mathrm{m}^{1 / 2}$ [28].

The parameter $g_{c}$ is computed with the following relation (for plane strain):

$$
g_{c}=\left(1-v^{2}\right) \frac{K_{I C}^{2}}{E}
$$

\subsection{Adhesive properties}

There are several theories that describe the various mechanisms by which adhesion may occur $[30,25,6]$ such as electrostatic charge transfer, mechanical interlocking, molecular diffusion or chemical bonding. There are however some specific theories which have been proposed for ice adhesion, for example, the liquid-like layer theory, originally proposed by Faraday and later investigated by Weyl and Jellinek $[20,23,27]$.

On the other hand, Petrenko and Ryzhkin proposed an electrostatic ice adhesion theory [21]. They evaluated the adhesion energy per unit area by numerically solving the problem of minimization of potential electrostatic energy. The adhesion energies range from $0.08 \mathrm{~J} \mathrm{~m}^{-2}$ to $1.3 \mathrm{~J} \mathrm{~m}^{-2}$. These results are comparable, at least in the vicinity of the lower bound, to those obtained experimentally by Sonwalkar [33] using Raman spectroscopy.

As far as atmospheric ice is concerned, experimental studies exist in the literature [41,22]. However, the results are usually highly scattered.

In this study, the adhesion energy measured by Sonwalkar [33] for an ice/aluminium interface will be used: $w=0.097 \mathrm{~J} \mathrm{~m}^{2}$. So as to have a complete set of material parameters, the effective stiffness of the interfacial bonds, $k$, and the coefficient $\alpha_{\tau}$ remain to be determined.

Several experimental studies on ice adhesion exist $[5,30,42]$. However, it should first be noted that once again, the data are highly scattered. Moreover, these experiments usually measure the shear strength of adhesion using test apparatuses that are difficult to reproduce within the current modelling framework (involvement of a flexible substrate, three dimensional geometries, etc.). With respect to this issue, one experiment of interest is the parallel-plate shearing experiment of [14]. However, as already stated, there are two parameters that need identifying: $k$ and $\alpha_{\tau}$. Hence, the approach that is adopted here is to use the data from [14] to identify $k$ assuming $\alpha_{\tau}=1$ (same contribution for normal and tangential dis-

Table 1

Elastic constants of interest for isotropic polycrystalline ice at $T=-16^{\circ} \mathrm{C}$.

\begin{tabular}{ll} 
Young's modulus, $E$ & $9.33 \times 10^{9} \mathrm{~Pa}$ \\
Poisson's ratio, $v$ & 0.325 \\
\hline
\end{tabular}


placements). The effects of variations of $\alpha_{\tau}$ on the identified value of $k$ are also assessed. However, as will be discussed later, these variations will not affect the results of the ice shedding computations.

Identification of $k$ is done by numerically reproducing the parallel-plate shearing experiment of [14]. In this experiment, a $1.8 \mathrm{~mm}$ thick by $1.9 \mathrm{~cm}$ long ice block, adhering to a surface on the bottom, is sheared from its upper boundary until debonding occurs. The maximum shear load was measured to be $820 \mathrm{kPa}$. Hence, the parameter $k$ is identified so as to predict adhesive debonding for that given shear load (see Fig. 10).

The range within which $k$ is located is first narrowed down by dichotomy. The value of $k$ is then searched for using steps of $10^{14} \mathrm{~Pa} \mathrm{~m}^{-1}$. Fig. 11 shows the distribution of $\beta$ along the interface. It shows the local state of bondedness of the interface (a fully debonded interface corresponds to $\beta=1$ everywhere, as in Fig. 12). We see that for $k=8 \times 10^{14} \mathrm{~Pa} \mathrm{~m}^{-1}$ interfacial damage starts to build up but very quickly reaches a converged distribution. Therefore, this value of $k$ leads to a non debonded interface and is too strong.

Fig. 12 shows the propagation of interfacial damage as the iterative process advances and reaches a fully debonded state. Hence, the parameter $k$ identified by this method was found to be equal to $7 \times 10^{14} \mathrm{~Pa} \mathrm{~m}^{-1}$.

In addition, the effects of varying $\alpha_{\tau}$ were also tested. The values of $0.1,0.5$ and 2 were assigned to $\alpha_{\tau}$ which yielded different values of $k$ (to the chosen probing precision of $10^{14} \mathrm{~Pa} \mathrm{~m}^{-1}$ ), which are summed up in Table 2:

In Section 6, the effects of these variations on the ice shedding computation will be discussed.

\section{Numerical simulations}

Icing and ice protection are complex unsteady phenomena. Icing codes typically include several modules in order to determine water droplet catch efficiency, ice growth and heat and mass transfer. A full electrothermal de-icing numerical simulation requires going through all of these steps and adding a shedding criterion $[9,38,10,43,39]$. But this whole panel of modules shall not be used here. What is being examined is a very specific mechanism. The possibilities offered by numerical simulation are hence used to look into the effects of varying only some parameters, all others being fixed. Three cases are considered (described below). In these cases time is freezed: the ice shape is constant (within each case), the flow field is constant, and the length of the water film $L_{f}$ is increased (by increments of $5 \%$ of $L_{t}$ ) until the critical length leading to ice shedding is reached.

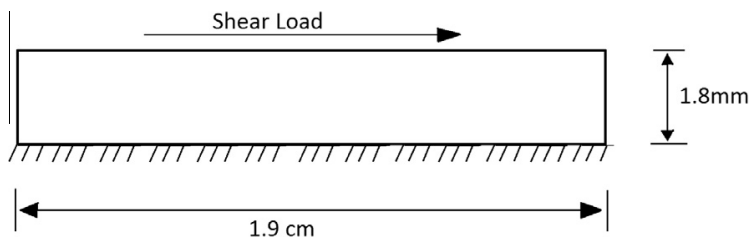

Fig. 10. Adhesive parallel plate shear test.

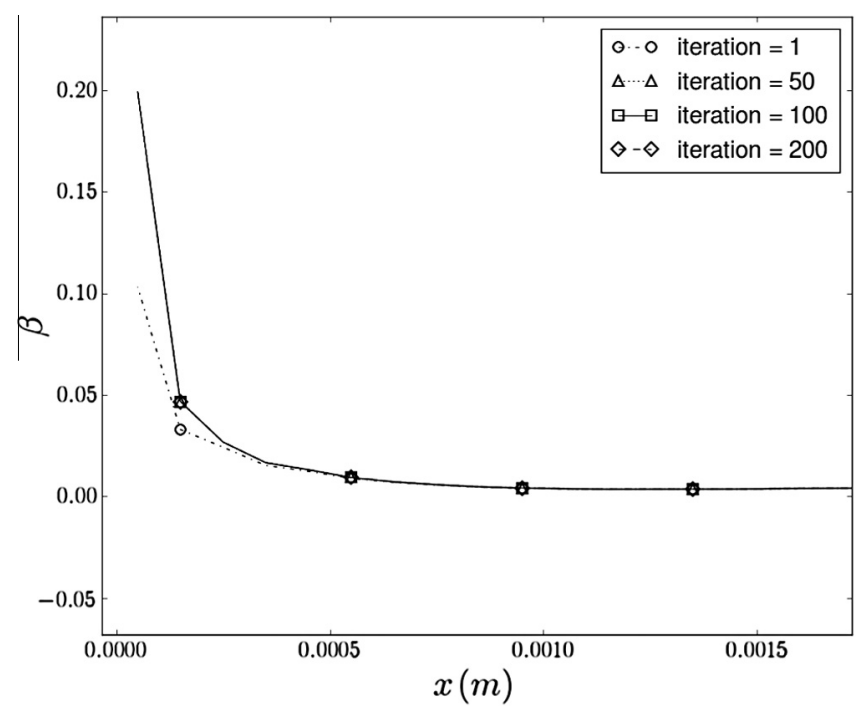

Fig. 11. Adhesive parallel plate shear test: non debonded interface for $k=8 \times 10^{14} \mathrm{~Pa} \mathrm{~m}^{-1}$. 


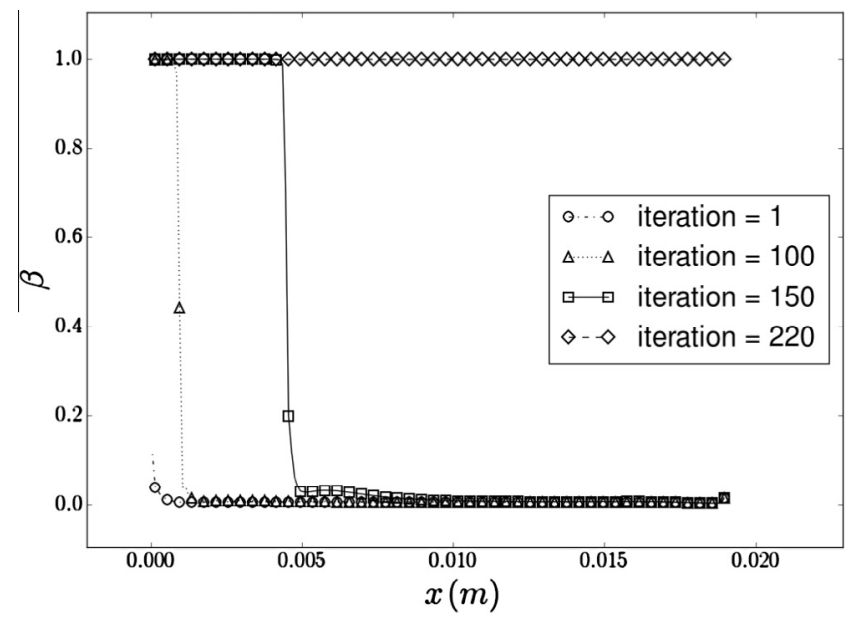

Fig. 12. Adhesive parallel plate shear test: fully debonded interface for $k=7 \times 10^{14} \mathrm{~Pa} \mathrm{~m}^{-1}$.

Table 2

Values of $k$ found for different values of $\alpha_{\tau}$.

\begin{tabular}{llll}
\hline$\alpha_{\tau}$ & 0.1 & 0.5 & 1 \\
$k\left(\mathrm{~Pa} \mathrm{~m}^{-1}\right)$ & $9 \times 10^{14}$ & $7 \times 10^{14}$ & 2 \\
\hline
\end{tabular}

Table 3

Aerodynamic conditions.

\begin{tabular}{llll}
\hline Mach & $P_{\infty}(\mathrm{Pa})$ & $T_{\infty}(\mathrm{K})$ & $\alpha\left(^{\circ}\right)$ \\
\hline 0.4 & 61640.0 & 263.15 & 2.0 \\
\hline
\end{tabular}

The numerical experiments are defined by using two elements:

- An airfoil: here a NACA0012 airfoil is used.

- A generic ice shape: it is chosen to be shaped as a teardrop. It represents a simple form of intercycle ice shape. Its characteristic thickness is noted $h_{\text {ice }}$ and is set to $1.0 \mathrm{~mm}, 3.0 \mathrm{~mm}$ or $5.0 \mathrm{~mm}$, depending on the case.

In typical icing conditions, ice will build up along the whole wingspan. Therefore, in this case, the plane strain two dimensional elasticity formulation retained for it corresponds to the case of a body of infinite span. Porosity is set to $3 \%$ for all cases and grain size to $d_{\text {grain }}=7 \mathrm{~mm}$, which are typical values observed for atmospheric ice [28].

The ice shape is placed on the aerodynamic shape. A location that is coherent with the actual functioning of an ETIPS is chosen (not far from the parting strip).

The aerodynamic conditions are given in Table 3. The commercial code ANSYS Fluent [16] is used to compute the aerodynamic flow field, and more particularly the pressure distribution on the ice block. This pressure distribution will define the Neumann boundary conditions for the crack propagation problem. Fig. 14 shows a global view of the problem. Fig. 13 is a generic sketch where we have illustrated the three different boundary conditions:

$$
\begin{aligned}
\sigma \cdot n+k F(\beta, u) u=\beta p_{\text {redistributed }} \cdot n \text { on } \Gamma_{u} \\
\sigma \cdot n=p_{\text {exterior }} \cdot n \text { on } \Gamma_{\text {exterior }} \\
\sigma \cdot n=p_{\text {redistributed }} \cdot n \text { on } \Gamma_{p}
\end{aligned}
$$

To summarize, three numerical experiments are performed for three increasing thicknesses of the ice shape. The numerical experiment consists in varying $L_{f}$ until fracture occurs, starting with $L_{f}=0$. Information on the mesh and spreading length are summed up in Appendix A.

\section{1. $1 \mathrm{~mm}$ ice thickness}

This case consists in the thinnest of the three ice shapes that are considered. Hence, it is also the case which presents the lowest pressure decrease on its outer surface. The pressure distribution on this ice shape is shown in Fig. 15. The maximum pressure differential is of about $5 \mathrm{kPa}$. 


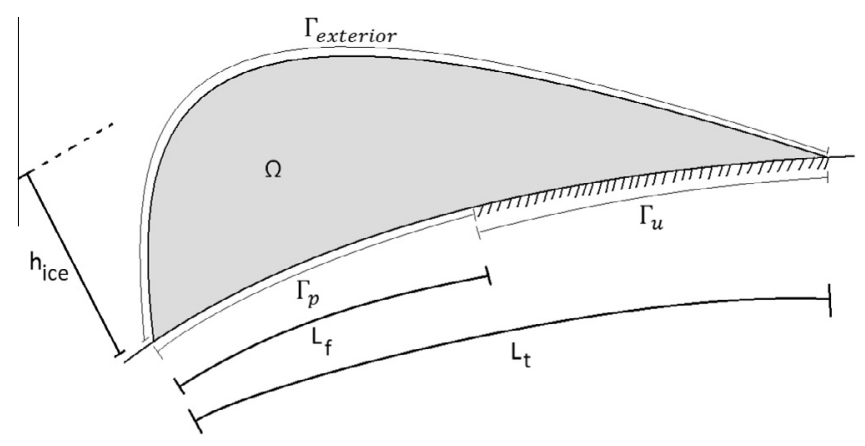

Fig. 13. Boundary and length definitions.

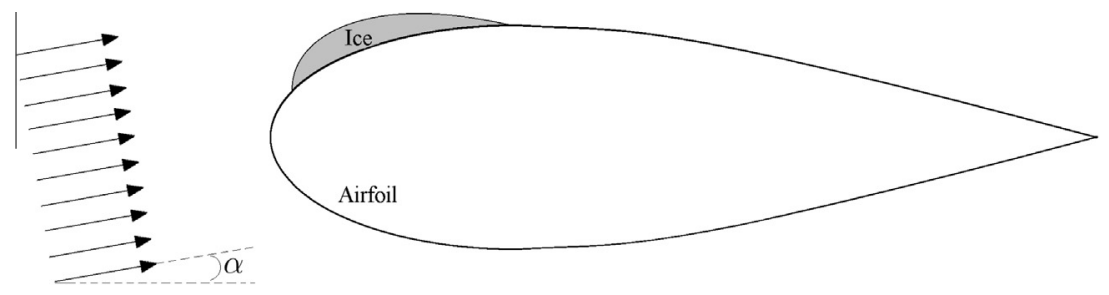

Fig. 14. Sketch of the case.

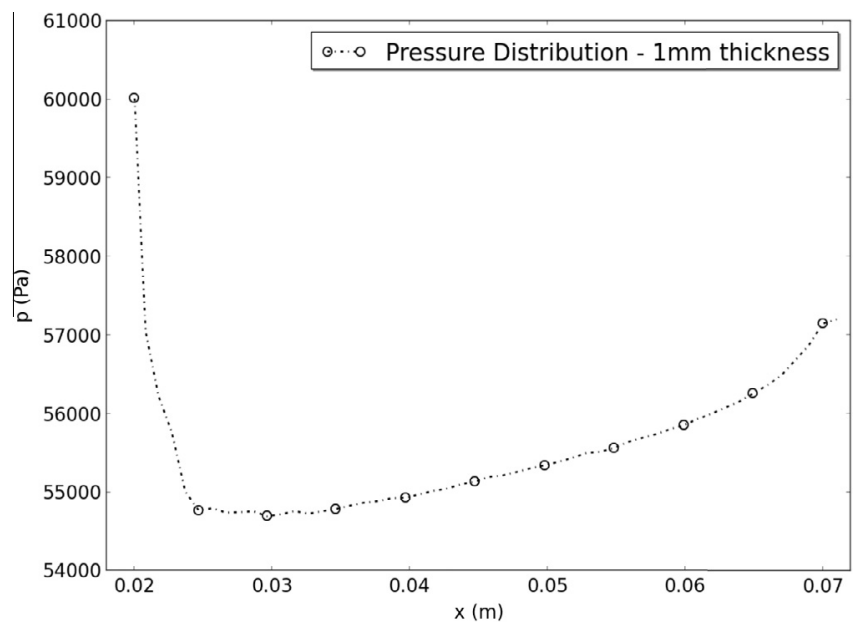

Fig. 15. Pressure distribution on the $1 \mathrm{~mm}$ thick ice lump.

The critical melted length leading to ice shedding was found to be $L_{f}=15 \% L_{t}$. The shedding mechanism starts with adhesive debonding over a short distance, as shown in Fig. 16.

Then, the critical brittle failure length is reached, leading to the nucleation and propagation of a crack through the ice thickness, as can be seen in Fig. 17. In this case the critical bulk failure length is equal to $20 \% L_{t}$. Note that without activating the debonding model, ice shedding would be predicted by brittle bulk failure for $L_{f}=20 \% L_{t}$.

\section{2. $3 \mathrm{~mm}$ ice thickness}

This case consists in a $3 \mathrm{~mm}$ thick ice shape. As could be expected by the increased thickness, the pressure distribution shows (Figs. 18 and 20) a stronger decrease on the outer surface of the lump. The maximum pressure differential is of $14 \mathrm{kPa}$ (see Fig. 20).

However, due to the larger thickness, the ice lump has a higher resistance to deformation. Therefore, the critical melted length in this case is $L_{f}=30 \% L_{t}$.

Also, adhesive debonding extends over a higher length until reaching the critical brittle failure length, which is here of $L_{b}=40 \% L_{t}$ (see Fig. 19). 


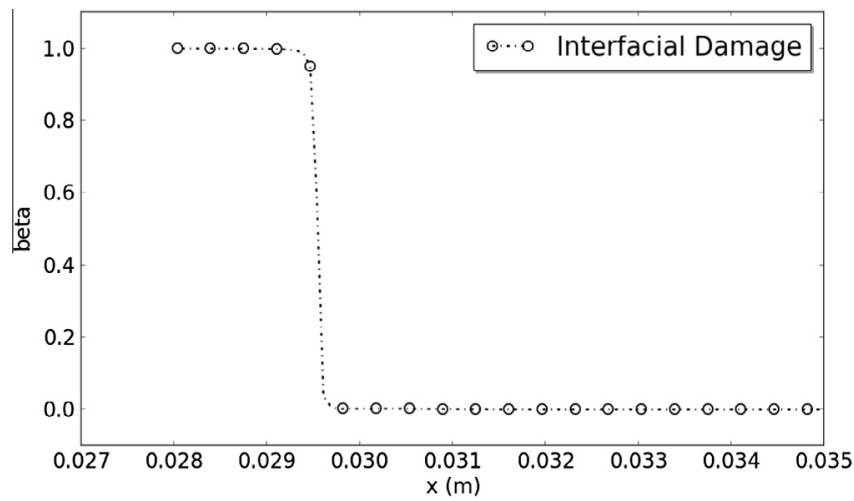

Fig. 16. $1 \mathrm{~mm}$ thickness case: interfacial damage distribution before brittle failure.

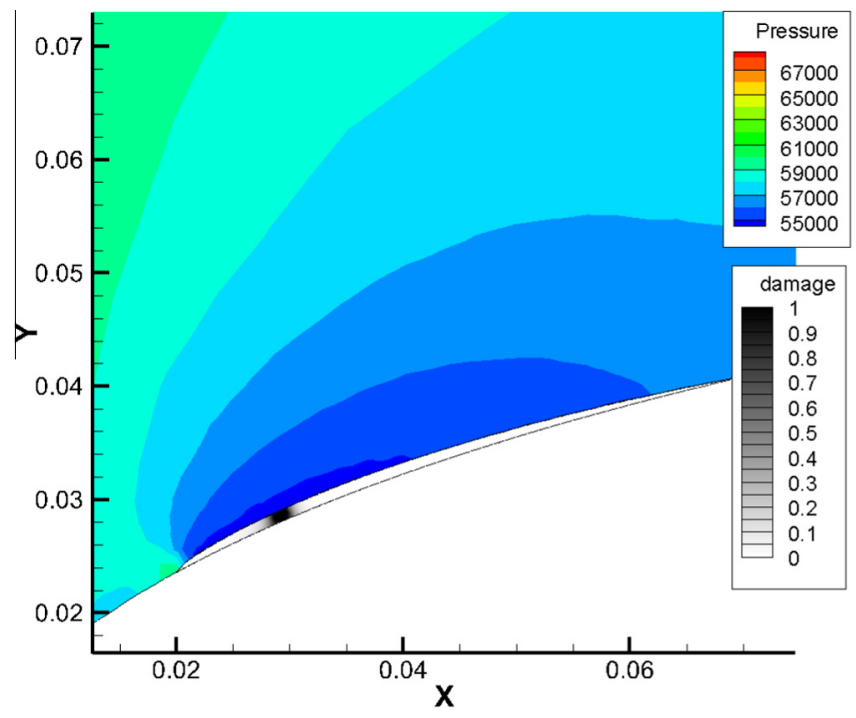

Fig. 17. $1 \mathrm{~mm}$ thickness case: bulk damage field and pressure field.

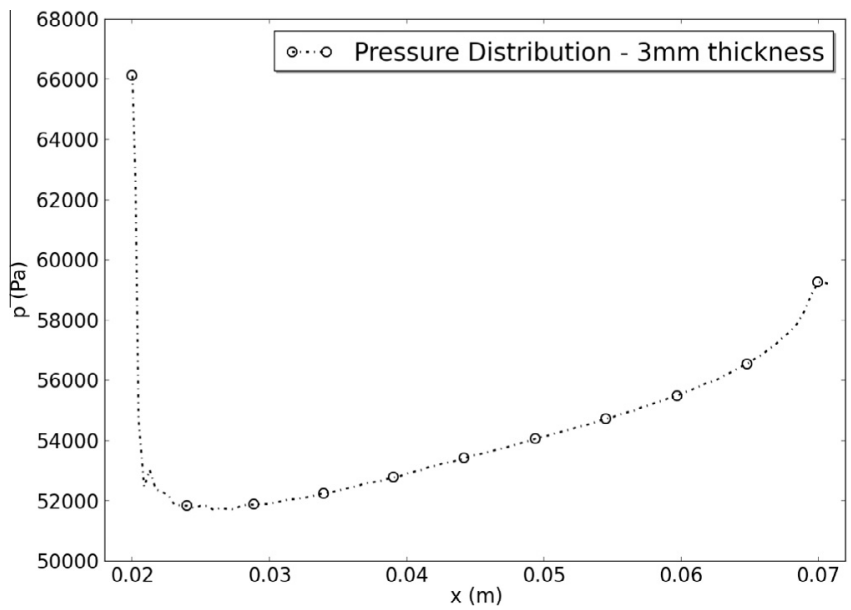

Fig. 18. Pressure distribution on the $3 \mathrm{~mm}$ thick ice lump. 


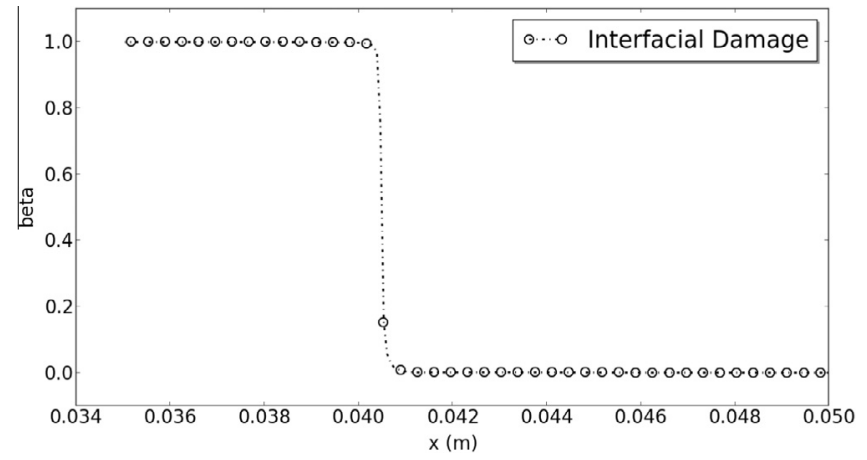

Fig. 19. $3 \mathrm{~mm}$ thickness case: interfacial damage distribution before brittle failure.

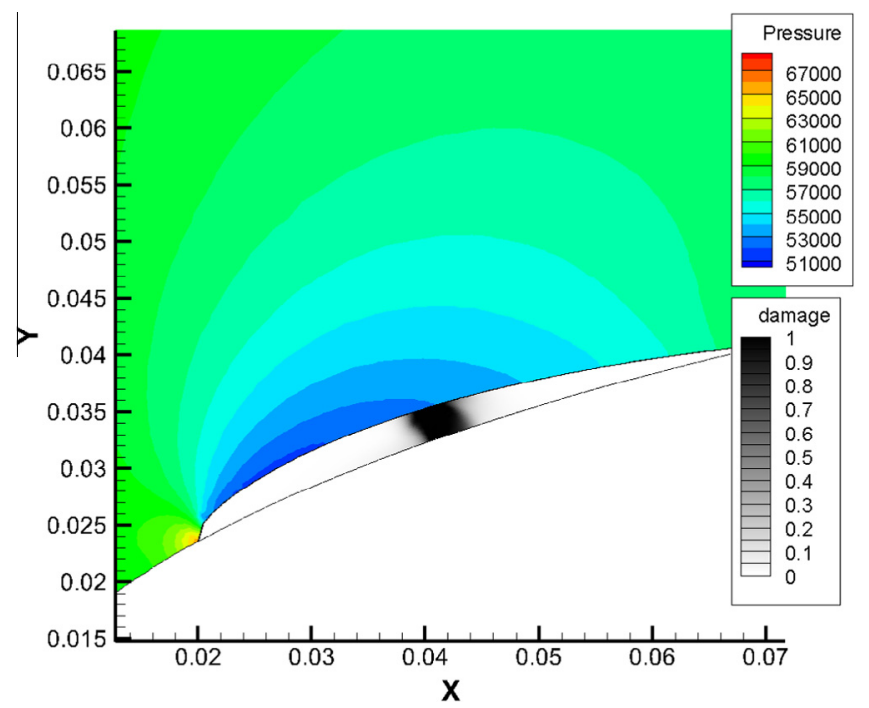

Fig. 20. $3 \mathrm{~mm}$ thickness case: bulk damage field and pressure field.

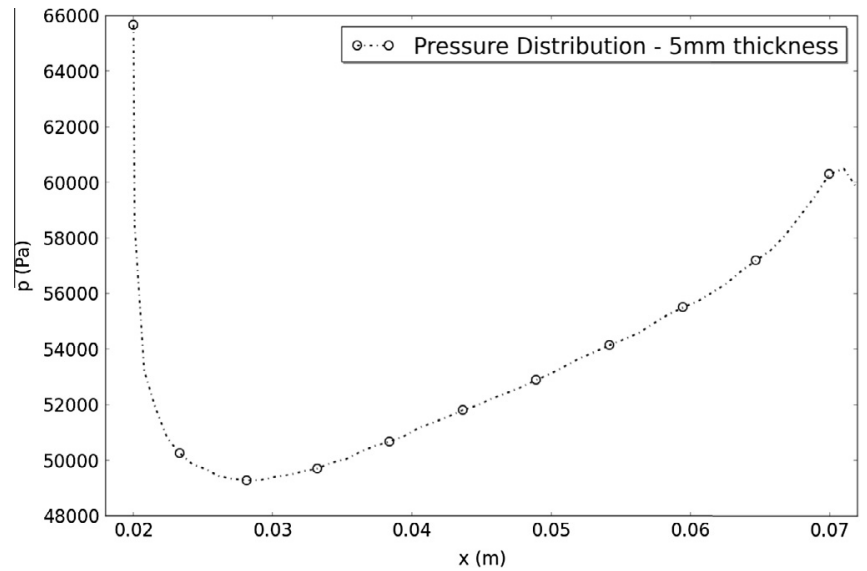

Fig. 21. Pressure distribution on the $5 \mathrm{~mm}$ thick ice lump.

\section{3. $5 \mathrm{~mm}$ ice thickness}

The final case is the one having the largest thickness. This yields an even larger pressure drop on the outer surface of the lump. The pressure distribution on the external surface is shown in Fig. 21. The maximum pressure differential is of about $17 \mathrm{kPa}$. 


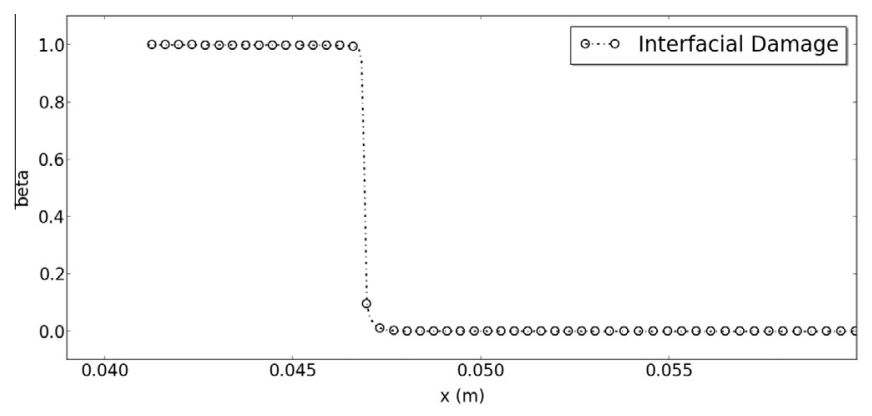

Fig. 22. $5 \mathrm{~mm}$ thickness case: interfacial damage distribution before brittle failure.

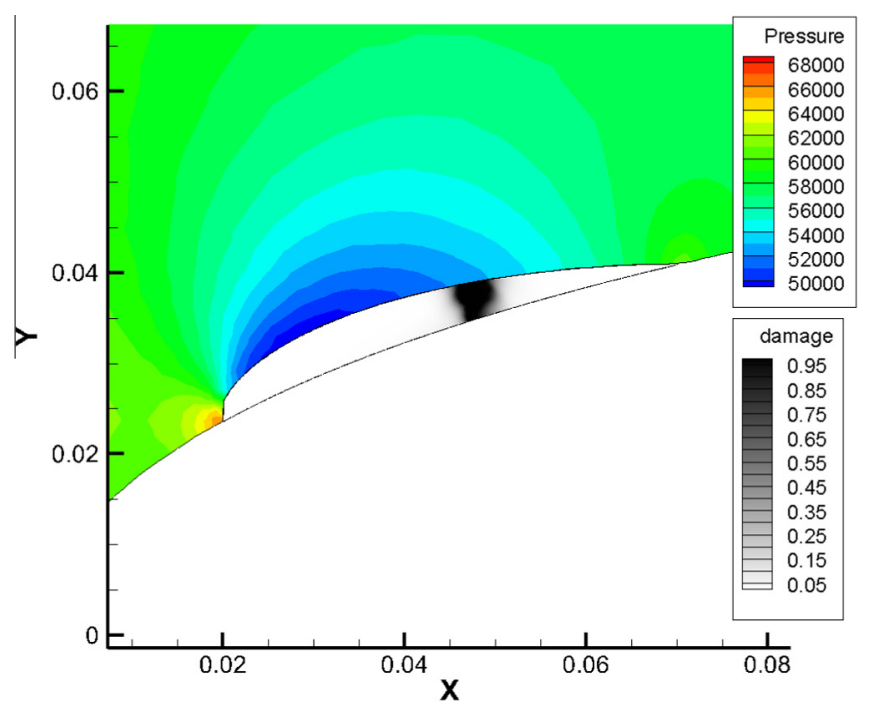

Fig. 23. $5 \mathrm{~mm}$ thickness case: bulk damage field and pressure field.

Coherently with the trend observed when going from a thickness of $1 \mathrm{~mm}$ to one of $3 \mathrm{~mm}$, the critical melted length is increased. In this case it is found to be of $L_{f}=40 \% L_{t}$.

Here, the adhesive debonding extends over approximately the same distance as for the $3 \mathrm{~mm}$ case (see Fig. 22). The critical brittle failure length is of $L_{b}=55 \% L_{t}$. The cracked state for this case is shown in Fig. 23.

\subsection{Surface Tension Effects}

In all the previous cases, surface tension effects have not been considered. In fact, when the liquid water film forms at the interface between the ice block and the surface a contact angle appears due to surface tension (as illustrated in Fig. 24). ${ }^{2}$ The value of this angle will depend on the physical properties of the interface between water and the surface.

The pressure difference across the interface is linked to the contact angle $\theta_{c}$, the liquid film thickness $h_{f}$ and the water-air surface tension $\gamma_{s t}\left(\simeq 70 \mathrm{mN} \mathrm{m}^{-1}\right)$ through the Young-Laplace equation:

$$
\Delta p=p_{1}-p_{2}=\frac{\gamma_{s t}}{h_{f} / 2} \cos \left(\theta_{c}\right)
$$

This means that for the previous cases, a contact angle of $\pi / 2$ was implicitly assumed. However, for low values of $h_{f}$ and contact angles approaching 0 (superhydrophilic) or $\pi$ (superhydrophobic), surface tension effects may be non negligible. Moreover, in the case of a hydrophilic surface the pressure inside the water film will be lower than the exterior air pressure. This means that a very hydrophilic surface may lower the ability of the aerodynamic forces to detach the ice block in the manner described previously. On the other hand, a very hydrophobic surface will have the opposite effect, and could enhance the previously described mechanisms.

\footnotetext{
${ }^{2}$ In fact, the liquid water/ice and liquid water/surface interfaces have different physical characteristics. Therefore, the contact angle will not be the same for the two interfaces. However, this discussion of surface tension effects is qualitative. The two contact angles will therefore be considered equal.
} 


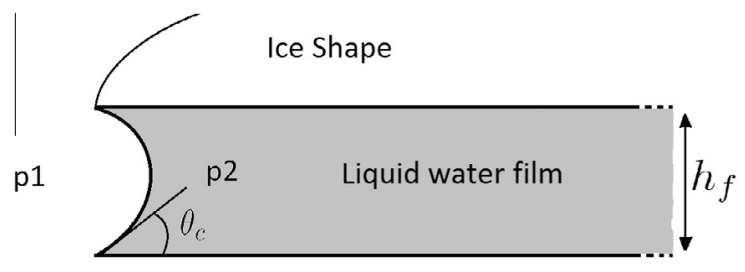

Fig. 24. Illustration of the contact angle.

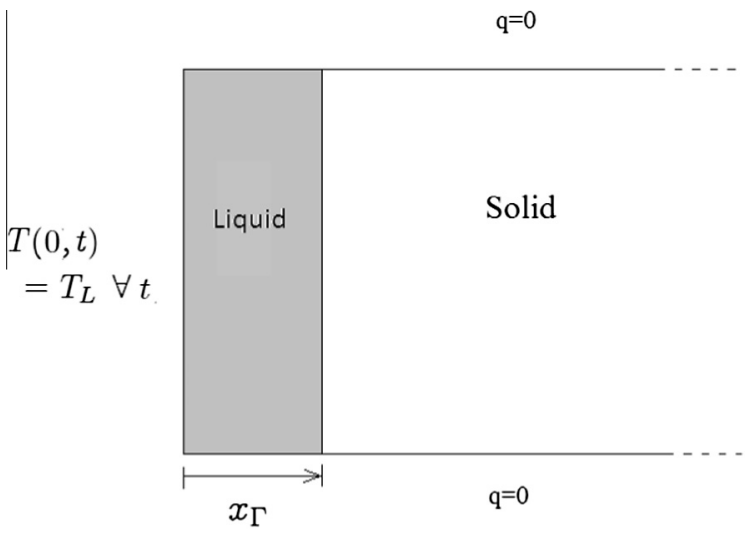

Fig. 25. Illustration of the Stephan problem.

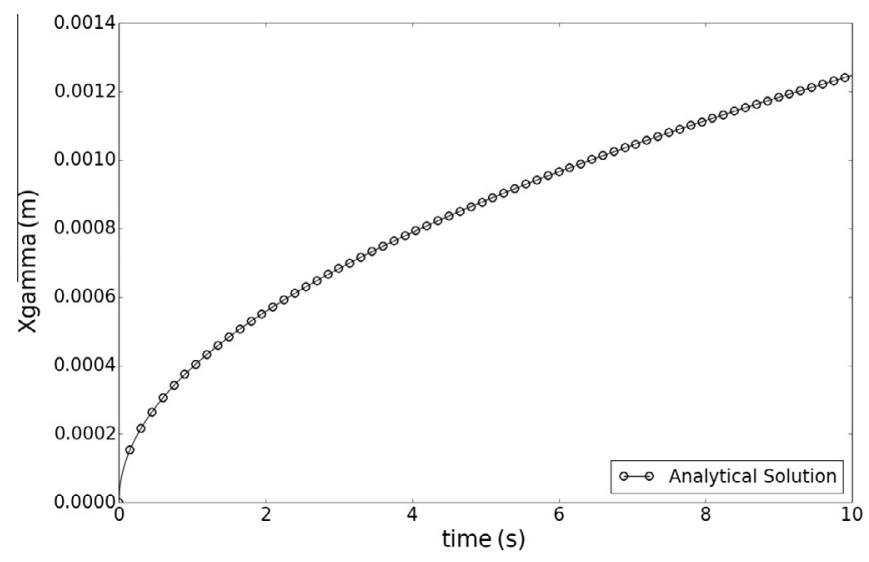

Fig. 26. Melting front position obtained by solving the Stephan problem.

In order to investigate this issue a little further, it is interesting to try to give an estimate of the liquid film thickness in the context of ice protection systems. This can be achieved by considering the Stephan problem (illustrated in Fig. 25). Consider a slab of ice, at an initial temperature $T_{i}<T_{m}$ (where $T_{m}$ is the melting temperature). The left boundary of the slab is maintained at a temperature $T_{L}>T_{m}$ which has the effect to melt it. A melting front, noted $x_{\Gamma}$, then propagates through the ice (see Fig. 26).

It can be shown that the position of the melting front follows the equation [4]:

$$
x_{\Gamma}(t)=2 \chi \sqrt{\alpha_{l} t}
$$

where $\alpha_{l}$ is the thermal diffusivity of liquid water and $\chi$ is a coefficient obtained by solving an equation linked to the given data of the problem.

For example, for $T_{L}=340 \mathrm{~K}$ and $T_{i}=260 \mathrm{~K}$ (which is representative of ice protection systems), the following solution is obtained: 
Hence, if the de-icing system has only been activated for a few seconds (1-5 s), the liquid water film will have a thickness of the order of $10^{-4} \mathrm{~m}$. For the sake of fixing some values, let's consider that the system has been activated for approximately $2 \mathrm{~s}$, yielding $h_{f}=0.0005 \mathrm{~m}$. In the case of an ideally superhydrophilic surface, $\cos \left(\theta_{c}\right)=1$ and therefore $p_{1}-p_{2}$ will be of about $280 \mathrm{~Pa}$. Conversely, in the case of an ideally superhydrophobic surface $\left(\cos \left(\theta_{c}\right)=-1\right) p_{1}-p_{2}$ will be of about $-280 \mathrm{~Pa}$. This pressure difference has to be compared with the maximum pressure differential induced by the aerodynamic flow, which is of the order of $10 \mathrm{kPa}$. Hence, in this case, surface tension effects may have some effect but only slightly.

On the other hand, consider a very low activation time which would yield $h_{f}=0.0001 \mathrm{~m}$. In this case $\Delta p= \pm 1.4 \mathrm{kPa}$ (depending on the nature of the surface). Hence, for these low activation times, leading to low liquid water film thicknesses, surface tension may have a visible effect, either positive (hydrophobic surface) or negative (hydrophilic surface).

\section{Discussion}

The results of the numerical experiments show that a phenomenon of detachment before complete melting of the interface is possible. At a given critical value of $\frac{L_{f}}{L_{t}}$, the stress concentration is sufficient to initiate adhesive debonding. This debonding propagates until a critical brittle failure length $L_{b}$ is reached. At this point crack nucleation and propagation over the whole thickness occurs, therefore tearing off a certain amount of ice.

It is also interesting to note the effect of increasing the thickness of the ice block. Indeed, as the pressure decrease is more pronounced for larger thickness, it could have been expected that increasing the thickness leads to a lower critical melted length. The simulations show that it is the opposite that happens. This can be explained in the following way. An ice block with increased thickness will be more resistant to deformation. The pressure decrease created by the increased thickness is not sufficient to counterbalance the increase in resistance of the block. Therefore, the critical melted length increases with increasing thickness.

Moreover, when lowering the thickness of the block, it seems that the length over which extends the adhesive debonding process is reduced. This effect can clearly be seen by comparing the case of $1 \mathrm{~mm}$ thickness (see Fig. 16) to that of $3 \mathrm{~mm}$ (see Fig. 19). Nevertheless, this trend also appears to have a certain limit. Indeed, the length of adhesive debonding between the $3 \mathrm{~mm}$ case and the $5 \mathrm{~mm}$ case is almost equal.

It is also interesting to discuss some of the hypotheses that were made and their possible effects:

- Empirical laws were used in order to characterize the mechanical behaviour of atmospheric ice which have their limitations. It is very complicated to characterize the mechanical properties of atmospheric ice. Most of the time experimental results are highly scattered and no precise laws are available. Hence the reader should bear in mind that the mechanisms discussed here are presented more from a qualitative standpoint.

- The ice shape may grow in time. However this should not impact the results significantly. The characteristic time of adhesive debonding and crack propagation is much lower than that of ice accretion (as soon as the critical ice shedding conditions are reached).

- In reality, a feedback process between the lifting of the ice shape and the flow field occurs. The lifting of the ice shape occurs simultaneously with a corresponding change in the flow field. And this change in flow field immediately induces a new lifting force on the ice shape. This process is not taken into account. We consider the pressure distribution given by the first computation as fixed. Still, the change in pressure distribution would lead to a higher uplifting force in this case. Therefore this should not impact the results. Moreover, experimental observation shows that ice shedding occurs almost instantaneously, hence leaving little time for such a process to have a significant impact.

Still, the proposed mechanisms constitute an interesting way forward for future investigation and modelling of ice shedding. It should be borne in mind that the mechanisms conducive to ice shedding are not at all well understood. The mechanism presented here could provide quantitative and qualitative explanations to experimental observations made when testing de-icing systems in icing wind tunnels. Moreover, and maybe more importantly, this mechanism could provide an interesting path for experimental investigations.

In addition, as discussed in Section 4.2, the identification of all the adhesive physical parameters is a difficult issue. The ice shedding computation of the $3 \mathrm{~mm}$ thickness case was performed with other values of $k\left(6 \times 10^{14} \mathrm{~Pa} \mathrm{~m}^{-1}\right.$ and $8 \times 10^{14} \mathrm{~Pa} \mathrm{~m}^{-1}$ ). However, it was found that these variations do not impact the obtained results. This means that these variations are below the precision threshold of the chosen control parameters (steps of $5 \%$ in $L_{f}$ ).

\section{Conclusion}

In this paper, a coupled quasi-static adhesion/brittle failure model has been presented and used to study a possible ice shedding mechanism. This mechanism is based on hydrostatic pressure equilibrium between the external flow and the water film forming at the interface between the ice and the aeronautical surface. This recovered pressure creates a lifting force which acts to detach the ice from the surface. 
The modelling and numerical strategy were presented and some aspects concerning the boundedness of the bulk damage variable $d$ were discussed.

In order to study this mechanism, three numerical experiments are considered. All of them showed that the ice shedding mechanism based on the hydrostatic pressure equilibrium is relevant. In all cases, the detachment process starts with adhesive debonding until a critical brittle failure length is reached. At that point a crack nucleates and propagates through the thickness of the ice block. This result is in qualitative agreement with some of the observations of Wei et al. [48].

The effects of change in thickness were investigated, showing that an increase in thickness leads to an increase in the critical melted length, despite the higher pressure differential. Moreover, for the lower thickness ice block, it seems that the debonding process plays a less important role than for blocks with larger thickness.

Surface tension effects were also briefly discussed. Indeed, depending on the nature of the surface, surface tension effects could have a visible effect. The detachment process could be enhanced by the use of a hydrophobic coating. This aspect is already under considerable research (and has been for many years). Moreover, thermo-elastic effects due to temperature gradients, could also be at play, as suggested by the thermal shock experiments of King and Fletcher $[44,45]$. This point is the subject of current work.

In view of the lack of precise experimentation on this particular issue, it is difficult to provide quantitative conclusions at this time. The goal here was more to investigate a particular ice shedding mechanism. More experimental data are now needed so as to characterize atmospheric ice more precisely and provide more accurate constitutive laws, which is a highly challenging issue.

Finally, the simulations performed in this study, showing that the proposed mechanisms are relevant, could be used to guide experimental investigations specific to ice shedding. Once more data will have been gathered on this difficult issue, it will be possible to identify the parameters of the proposed models with greater precision. This will enable quantitative predictions and realistic simulations of ice protection systems.

\section{Acknowledgements}

Part of this work was performed under an ANRT/Airbus C.I.F.R.E grant. We gratefully acknowledge their support.

\section{Appendix A. Numerical settings}

All adhesion/brittle failure computations were realized with an in-house finite element code. Triangular elements were used and the meshes were generated using GMSH [11]. Higher order elements could be used; however, this appears to be unnecessary for the purpose of this study. The numerical settings are summed up in Table A.4:

The aerodynamic computations were done using ANSYS Fluent [16]. All meshes consisted of triangular elements $(\sim 35,000)$.

\section{Appendix B. Computation of the projection operators}

This appendix describes the computation of the projection operators used for the bulk damage model. This is a classic result of tensor calculus (see for example [12]). As seen in Section 3.1 the computation of the elastic energy is based on the following splitting of strain into tensile and compressive parts:

$$
\epsilon=\epsilon_{+}+\epsilon_{-}
$$

Differentiating this relation with respect to $\epsilon$ yields the following:

$$
\mathbb{1}=\frac{\partial \epsilon_{+}}{\partial \epsilon}+\frac{\partial \epsilon_{-}}{\partial \epsilon}=\mathbb{P}^{+}+\mathbb{P}^{-}
$$

And therefore the splitting of $\epsilon$ may be rewritten as follows:

$$
\epsilon=\underbrace{\mathbb{P}^{+}}_{\partial_{\epsilon} \epsilon_{+}}: \epsilon+\underbrace{\mathbb{P}^{-}}_{\partial_{\epsilon} \epsilon_{-}}: \epsilon
$$

Thus, the tensile and compressive components of strain are linked to the strain tensor itself by the use of projection operators, here noted $\mathbb{P}^{+}$and $\mathbb{P}^{-}$, through relations (B.2) and (B.3).

Table A.4

Numerical settings.

\begin{tabular}{lcll}
\hline$\varnothing$ & Number of elements & Type of element & $l$ \\
\hline$k$ identification & 8000 & Lagrange P1 & $5.0 \times 10^{-4}$ \\
$1 \mathrm{~mm}$ thickness & 16,000 & Lagrange P1 & $5.0 \times 10^{-4}$ \\
$3 \mathrm{~mm}$ thickness & 50,000 & Lagrange P1 & $5.0 \times 10^{-4}$ \\
$5 \mathrm{~mm}$ thickness & 80,000 & Lagrange P1 & $5.0 \times 10^{-4}$ \\
\hline
\end{tabular}




$$
\begin{aligned}
& \epsilon_{+}=\mathbb{P}^{+}(\epsilon): \epsilon \\
& \epsilon_{-}=\mathbb{P}^{-}(\epsilon): \epsilon
\end{aligned}
$$

These projection operators are fourth order tensors whose computation requires the eigenvalues and eigenvectors of $\epsilon$. As can be seen in Eq. (B.1), the projection operators are partial derivatives of tensor valued tensor functions. They are computed using the following classic tensor calculus formula (see $[12,35]$ ).

$$
\frac{\partial G(B)}{\partial B}_{i j k l}=\sum_{a} \sum_{b} \frac{\partial g_{a}}{\partial \lambda_{b}} n_{a, i} n_{a, j} n_{b, k} n_{b, l}+\sum_{a} \sum_{b \neq a} \frac{1}{2} \frac{g_{a}-g_{b}}{\lambda_{a}-\lambda_{b}} n_{a, i} n_{b, j}\left(n_{a, k} n_{b, l}+n_{b, k} n_{a, l}\right)
$$

where $G$ represents the tensor valued tensor function, $B$ is the argument tensor, $\lambda_{a}$ are the eigenvalues, $n_{a}$ are the eigenvectors, $g_{a}$ are the diagonal functions associated with the representation of $G(B)$ in its eigenbasis. Note that this means that $G(B)$ has the same eigenvectors as $B$, which is the case here for the positive-negative part decomposition. Here, $g_{a}$ is either $\left\langle\lambda_{a}\right\rangle_{+}$or $\left\langle\lambda_{a}\right\rangle_{-}$(depending on the projector of interest), which are the eigenvalues of the positive/negative parts.

As the chosen shape functions is Lagrange P1, the strain tensor is constant on each cell of the mesh. On a given cell $K$, the characteristic polynomial of the two dimensional strain tensor has the well known form:

$$
\chi=X^{2}-\operatorname{tr}\left(\epsilon_{K}\right) X+\operatorname{det}\left(\epsilon_{K}\right)
$$

The discriminant of this polynomial, $\Delta=\operatorname{tr}\left(\epsilon_{K}\right)^{2}-4 \operatorname{det}\left(\epsilon_{K}\right)=\left(\epsilon_{11, K}-\epsilon_{22, K}\right)^{2}+4 \epsilon_{12, K}^{2}$, is always positive. The eigenvalues are therefore real and given by:

$$
\epsilon_{1 / 2, K}=\frac{1}{2}\left(\operatorname{tr}\left(\epsilon_{K}\right) \pm \sqrt{\Delta}\right)
$$

The associated eigenvectors may also be computed using standard methods of linear algebra and are easy to compute analytically in our case. In the case where $\epsilon_{12} \neq 0$ the eigenvectors read:

$$
\boldsymbol{V}_{\mathbf{1}}=\left[\begin{array}{c}
\frac{\epsilon_{12}}{\epsilon_{1}-\epsilon_{11}} \\
1
\end{array}\right] \quad \boldsymbol{V}_{\mathbf{2}}=\left[\begin{array}{c}
\frac{\epsilon_{12}}{\epsilon_{2}-\epsilon_{11}} \\
1
\end{array}\right]
$$

These eigenvectors are then normalized. Note that in practice, to avoid floating point errors, it is checked whether $\left|\epsilon_{12}\right|>1 \mathrm{E}-16$ in order to use relations (B.7) and (B.4).

On the other hand, if $\epsilon_{12}=0$ (in practice $\left|\epsilon_{12}\right|<1 \mathrm{E}-16$ ), then the projectors reduce to a trivial form which can be explicitly treated. Indeed, for example if $\epsilon_{11} \geqslant 0$ and $\epsilon_{22} \geqslant 0$ the positive projector is simply given by

$$
\mathbb{P}_{1111}^{+}=1 ; \mathbb{P}_{2222}^{+}=1 ; \mathbb{P}_{i j k l}^{+}=0 \text { for all other } i, j, k, l
$$

Finally, note that the double eigenvalue case is included within the case $\epsilon_{12}=0$.

\section{Appendix C. Boundedness of the damage variables}

This section discusses numerical aspects regarding the boundedness of the bulk damage variable. The elements are always triangles and their nodes are noted $a_{i}$ (see Fig. C.28). Thanks to the shape functions, a function of interest can be represented locally over a given cell. This leads to the classic Lagrange P1 finite element solution procedure and it will therefore not be detailed further. The modelling strategy presented previously is based on the introduction of a damage variable $d$ which is required to vary between 0 and 1 . From a continuous standpoint, it is easy to show that the boundedness of $d$ follows from the maximum principle [19]. However, one question that may be asked is the following: does the numerical method guarantee the boundedness of $d$ ? Or under what conditions?

From a discretized point of view $d$ is the solution of the linear system:

$$
A_{d} d=R_{d}
$$

In order to solve this problem, one may use the Jacobi method (note that the Jacobi method is used for the proof only, and not to solve the system in practice). As the previous linear system has a unique solution, a Jacobi algorithm applied to it, if it converges, would lead to the same solution. It is based on the decomposition of $A_{d}$ into the sum of a diagonal, lower and upper part: $A_{d}=D_{d}+L_{d}+U_{d}$. The solution is then obtained via the following iterative algorithm:

$$
D_{d} d^{k+1}=R_{d}-U_{d} d^{k}-L_{d} d^{k}
$$

The matrix $A_{d}$ is the sum of a stiffness matrix $S_{d}$ and a matrix $M_{d}$ (resulting from the discretization of the term in $d$ ). Both these matrices are assembled from elementary matrices computed on each cell. For Lagrange P1 shape functions in two dimensions, they take the classical form as follows: 


$$
\begin{aligned}
& M_{d, K}=\left(\frac{g_{c}}{l}+2 \mathcal{H}\right)_{K}|K|\left[\begin{array}{ccc}
\frac{1}{6} & \frac{1}{12} & \frac{1}{12} \\
\frac{1}{12} & \frac{1}{6} & \frac{1}{12} \\
\frac{1}{12} & \frac{1}{12} & \frac{1}{6}
\end{array}\right] \\
& S_{d, K}=\frac{g_{c} l}{4|K|}\left[\begin{array}{ccc}
\left\|a_{2} a_{3}\right\|^{2} & \left(a_{2} a_{3} \mid a_{3} a_{1}\right) & \left(a_{2} a_{3} \mid a_{1} a_{2}\right) \\
\left(a_{2} a_{3} \mid a_{3} a_{1}\right) & \left\|a_{3} a_{1}\right\|^{2} & \left(a_{3} a_{1} \mid a_{1} a_{2}\right) \\
\left(a_{2} a_{3} \mid a_{1} a_{2}\right) & \left(a_{3} a_{1} \mid a_{1} a_{2}\right) & \left\|a_{1} a_{2}\right\|^{2}
\end{array}\right]
\end{aligned}
$$

where $\left(a_{i} a_{j} \mid a_{k} a_{l}\right)$ is the scalar product between the vectors linking $a_{i}$ to $a_{j}$ and $a_{k}$ to $a_{l} . K$ denotes the cell and $|K|$ its area.

Assuming the algorithm is initialized with $d^{0}=0$, the positivity of $d$ at subsequent iterations is guaranteed if all components of the diagonal matrix $D_{d}$ and right hand side $R_{d}$ are positive and all components of $L_{d}$ and $U_{d}$ are negative.

The right hand side is positive by definition of $\mathcal{H}$. Moreover, given the positivity of the diagonal terms of the elementary matrices, the diagonal matrix $D_{d}$ will also be positive.

However, the fact that all components of $L_{d}$ and $U_{d}$ are negative is not guaranteed a priori. First, the non diagonal terms of $M_{d, K}$ are positive. This issue may be overcome by using mass lumping, a standard procedure in finite element computation, which consists in concentrating all terms of the matrix $M_{d, K}$ onto its diagonal. The lumped matrix then has the following form:

$$
M_{d, K}^{\text {lumped }}=\left(\frac{g_{c}}{l}+2 \mathcal{H}\right)_{K}|K|\left[\begin{array}{ccc}
\frac{1}{3} & 0 & 0 \\
0 & \frac{1}{3} & 0 \\
0 & 0 & \frac{1}{3}
\end{array}\right]
$$

Second, the non diagonal terms of $S_{d, K}$ are not necessarily negative. Indeed, in the case of an element as illustrated in Fig. C.27, the scalar product $\left(a_{2} a_{3} \mid a_{3} a_{1}\right)$ will be positive.

Nevertheless, a good quality mesh will be composed of elements such as the one shown in Fig. C.28, thus leading to negative non-diagonal components.

Therefore, sufficient conditions to ensure the positivity of $d$ are to use a lumped matrix and a mesh whose elements do not present angles superior to $90^{\circ}$. A similar analysis can be performed for $d^{*}=1-d$ therefore leading to the boundedness of $d$ between values of 0 and 1 .

However, these conditions do not seem to be necessary for stability. In fact, tests were performed with a non-lumped mass matrix and bad quality elements (presenting one angle greater than $90^{\circ}$ ). Values of $d$ were observed to go out of

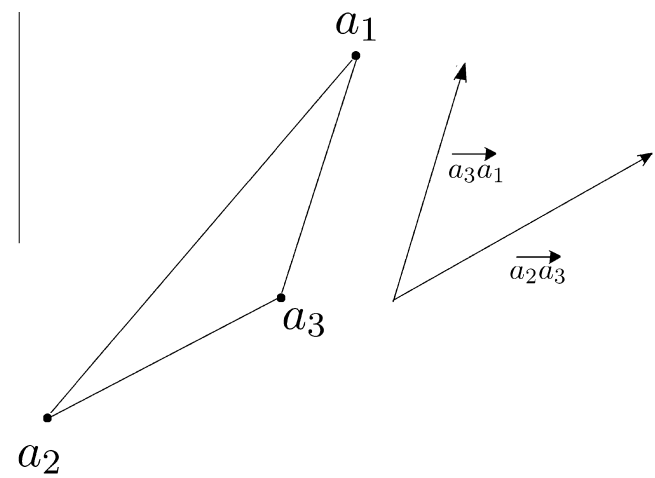

Fig. C.27. Case leading to a negative non-diagonal term of elementary stiffness matrix

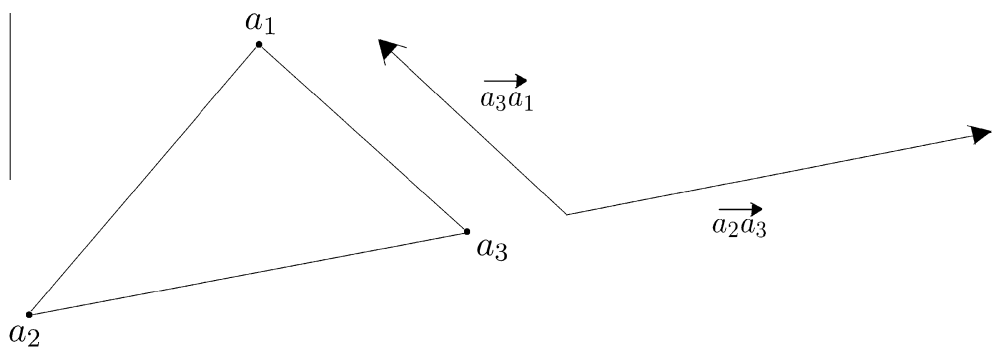

Fig. C.28. Case leading to a positive non-diagonal term of elementary stiffness matrix. 
the bounds $[0,1]$ only very slightly (effects to the third decimal). In addition, $d$ remained bounded between 0 and 1 when tests with a lumped matrix and a bad quality mesh were performed.

Note that concerning the adhesive damage variable $\beta$, boundedness is automatically respected by the algebraic relation (26).

\section{References}

[1] Aircraft Icing Handbook. Civil Aviation Authority; 2000.

[2] Zhang S, El Kerdi O, Khurram RA, Habashi WG. FEM analysis of in-flight ice break-up. Finite Elem Anal Des 2012;57:55-66.

[3] Griffith AA. The phenomena of rupture and flow in solids. Philos Trans Roy Soc Lond Ser A Containing Pap Math Phys Charact 1921;221:163-98.

[4] Solomon AD, Alexiades V. Mathematical modelling of melting and freezing processes. Hemisphere; 1993.

[5] Reich A, Scavuzzo R, Chu M. Survey of the mechanical properties of impact ice. In: 32nd aerospace sciences meeting and exhibit, Reno, NV, USA; 1994.

[6] Roche A. Les theories de l'adhesion et mesure de l'adherence. Le Vide, Les Couches Minces 1992;257:197-230.

[7] Mohamed AMA, Farzaneh M. An experimental study on the tensile properties of atmospheric ice. Cold Reg Sci Technol 2011;68:91-8.

[8] Bourdin B, Francfort GA, Marigo JM. The variational approach to fracture. J Elast 2008;91:5-148.

[9] Bennani L. Two dimensional modelling of electrothermal ice protection systems. PhD thesis. Institut Supérieur de l'Aéronautique et de l'Espace (ISAE); 2014.

[10] Bennani L, Villedieu P, Salaun M. Two dimensional model of an electro-thermal ice protection system. In: 5th AIAA atmospheric and space environments conference, San Diego, CA, USA, vol. 24; 2013.

[11] Geuzaine C, Remacle J-F. GMSH: a three-dimensional finite element mesh generator with built-in pre- and post-processing facilities. Int J Numer Meth Engng 2009;79(11):1309-31.

[12] Miehe C. Comparison of two algorithms for the computation of fourth-order isotropic tensor functions. Comput Struct 1998;66(1):37-43.

[13] Hofacker M, Miehe C, Welschinger F. A phase field model for rate-independent crack propagation: robust algorithmic implementation based on operator splits. Comput Methods Appl Mech Engng 2010;199(45):2765-78.

[14] Anderson DN, Reich AD. Tests of the performance of coatings for low ice adhesion. Technical memorandum 107399. NASA; 1997.

[15] Schulson EM, Duval P. Creep and fracture of ice. Cambridge University Press; 2009.

[16] Fluent. ANSYS. Fluent 6.3 documentation. Lebanon (NH): Fluent Inc.; 2006.

[17] Frémond M. Adhérence des solides. J Méc Théor Appl 1987;6(3):383-407.

[18] Francfort GA, Marigo JJ. Revisiting brittle fracture as an energy minimization problem. J Mech Phys Solids 1998;46(8):1319-42.

[19] Brezis H. Functional analysis, Sobolev spaces and partial differential equations. Springer; 2010.

[20] Jellinek HHG. Liquid-like (transition) layer on ice. J Colloid Interface Sci 1967;25(2):192-205.

[21] Ryzhkin IA, Petrenko VF. Physical mechanisms responsible for ice adhesion. J Phys Chem B 1997;101(32):6267-70.

[22] Druez J, Phan CL, Laforte JL, Nguyen DD. The adhesion of glaze and rime on aluminium electrical conductors. Trans CSME 1978-79;5(4).

[23] Jellinek HHG. Ice adhesion. Can J Phys 1962;40(10):1294-309.

[24] Bennani L, Villedieu P, Salaun M, Trontin P. Numerical simulation and modeling of ice shedding: process initiation. Comput Struct 2014;142:15-27.

[25] Shanahan MER. Adhesion and wetting: similarities and differences, vol. 205. United States: Rubber World; 1991 (1).

[26] Eskandarian M. Ice shedding from overhead electrical lines by mechanical breaking. PhD thesis. Université du Québec à Chicoutimi; 2005.

[27] Faraday M. Lecture given at the Royal Institution, London; June 1850

[28] Kermani M. Ice shedding from cables and conductors - a cracking model of atmospheric ice. PhD thesis. Universite du Quebec a Chicoutimi; 2007.

[29] Gagnon R, Kermani M, Farzaneh M. Bending strength and effective modulus of atmospheric ice. Cold Reg Sci Technol 2008;53(2):162-9.

[30] Freiberger A, Landy M. Studies of ice adhesion. J Colloid Interface Sci 1967;25(2):231-44.

[31] Raous M. Interface models coupling adhesion and friction. CR Méc 2011;339(7):491-501.

[32] Raous M, Cangémi L, Cocu M. A consistent model coupling adhesion, friction, and unilateral contact. Comput Methods Appl Mech Engng 1999;177 (3):383-99.

[33] Sonwalkar N. Molecular dynamics of ice-solid bi-material interfaces. PhD thesis. M.I.T.; 1992.

[34] Chau O, Fernández JR, Han W, Sofonea M. Variational and numerical analysis of a dynamic frictionless contact problem with adhesion. J Comput Appl Math 2003;156(1):127-57.

[35] Ogden RW. Non-linear elastic deformations. Chichester: Ellis Horwood; 1984.

[36] Gammon PH, Kiefte H, Clouter MJ, Denner WW. Elastic constants of artificial and natural ice samples by Brillouin spectroscopy. J Glaciol 1983;29 (103):433-60.

[37] Petrovic JJ. Review mechanical properties of ice and snow. J Mater Sci 2003;38(1):1-6.

[38] Chauvin R, Villedieu P, Trontin P. A robust coupling algorithm applied to thermal ice protection system unsteady modeling. In: 6th AIAA atmospheric and space environments conference-AVIATION 2014, Atlanta, GA, USA.

[39] Henry R. Development of an electrothermal de-icing/anti-icing model. In: AIAA 30th meeting, Reno, NV, USA.

[40] Scavuzzo R, Chu M, Ananthaswamy V. Influence of aerodynamic forces in ice shedding. In: 29th aerospace sciences meeting and exhibit, Reno, NV, USA; 1991.

[41] Scavuzzo RJ, Chu ML. Structural properties of impact ices accreted on aircraft structures. Technical report NASA contractor report 179580. The University of Akron; 1987.

[42] Sayward JM. Seeking low ice adhesion. Technical report. DTIC report 79-11; 1979

[43] Wright WB, Keith TG, De Witt KJ. Numerical simulation of icing, deicing and shedding. In: 29th aerospace sciences meeting, Reno, NV, USA; 1991.

[44] King WD, Fletcher NH. Thermal shock as an ice multiplication mechanism. Part I. Theory. J Atmos Sci 1976;33(1):85-96.

[45] King WD, Fletcher NH. Thermal shock as an ice multiplication mechanism. Part II. Experimental. J Atmos Sci 1976;33(1):97-102.

[46] Smith WL. Weather problems peculiar to the New York-Chicago Airway. Mon Weather Rev 1929;57:503-6.

[47] Leary WM. We freeze to please: a history of NASAs icing research tunnel and the quest for flight safety. The NASA History Series; 2002.

[48] Dempsey JP, Wei Y, Adamson RM. Ice/metal interfaces: fracture energy and fractography. J Mater Sci 1996;31(4):943-7. 